\title{
Spatial constraints on visual-tactile cross-modal distractor congruency effects
}

\author{
CHARLES SPENCE \\ University of Oxford, Oxford, England \\ FRANCESCO PAVANI \\ Università di Trento, Trento, Italy \\ and \\ JON DRIVER \\ University College London, London, England
}

\begin{abstract}
Across three experiments, participants made speeded elevation discrimination responses to vibrotactile targets presented to the thumb (held in a lower position) or the index finger (upper position) of either hand, while simultaneously trying to ignore visual distractors presented independently from either the same or a different elevation. Performance on the vibrotactile elevation discrimination task was slower and less accurate when the visual distractor was incongruent with the elevation of the vibrotactile target (e.g., a lower light during the presentation of an upper vibrotactile target to the index finger) than when they were congruent, showing that people cannot completely ignore vision when selectively attending to vibrotactile information. We investigated the attentional, temporal, and spatial modulation of these cross-modal congruency effects by manipulating the direction of endogenous tactile spatial attention, the stimulus onset asynchrony between target and distractor, and the spatial separation between the vibrotactile target, any visual distractors, and the participant's two hands within and across hemifields. Our results provide new insights into the spatiotemporal modulation of crossmodal congruency effects and highlight the utility of this paradigm for investigating the contributions of visual, tactile, and proprioceptive inputs to the multisensory representation of peripersonal space.
\end{abstract}

The external location of a tactile stimulus on the hand depends not only on which part of the hand has been stimulated, but also on where the hand lies in space. Representing this may involve the multisensory integration of visual, tactile, proprioceptive, and sometimes, even auditory cues regarding limb position (e.g., see Maravita, Spence, \& Driver, 2003, for a recent review). There has been increasing interest in how these various sensory cues may be weighted and integrated to enable people to localize tactile stimuli - to give rise to the felt position of our limbs and, ultimately, to the multisensory representation of peripersonal space (i.e., near the body) or extrapersonal space (e.g., see Rizzolatti, Fadiga, Fogassi, \& Gallese, 1997; Spence \& Driver, 2004). Research on these topics has expanded in recent years, with progress aided by converging findings from a number of different disciplines, including psychophysical and neuroimaging studies of normal participants, neuropsychological investigations of brain-damaged patients, and neurophysiological data from

Some of the results reported here were first presented at the 39th Annual Meeting of the Psychonomic Society in Dallas, Texas (see Spence, Pavani, \& Driver, 1998). Correspondence concerning this article should be addressed to C. Spence, Department of Experimental Psychology, University of Oxford, South Parks Road, Oxford OX1 3UD, England (e-mail: charles.spence@psy.ox.ac.uk). animals (see Graziano \& Botvinick, 2002; Làdavas, 2002; and Maravita et al., 2003, for reviews).

A behavioral task that has already proven useful in addressing some psychological issues regarding the multisensory representation of space in normal participants is the crossmodal distractor congruency task, first introduced by Spence, Pavani, and Driver in 1998. In a typical study, participants hold two foam blocks, one in either hand (see Figure 1). Embedded in the top and bottom of each foam block are vibrotactile stimulators and lightemitting diodes (LEDs). On each trial, a single vibrotactile target and a single visual distractor are each presented randomly (and independently) from any one of the four possible stimulus locations. Participants are required to make a speeded elevation (up/down) discrimination for each vibrotactile target stimulus, presented from the index finger or the thumb of either hand (i.e., above at the index finger or below at the thumb, in the posture typically used; see Figure 1), while simultaneously trying to ignore any visual distractor.

Although the visual distractors are just as likely to be presented from the same elevation as the vibrotactile targets as from different elevations, participants are typically significantly worse at discriminating the elevation of tactile targets when visual distractors are presented from an incongruent elevation (i.e., when the vibrotactile target is 


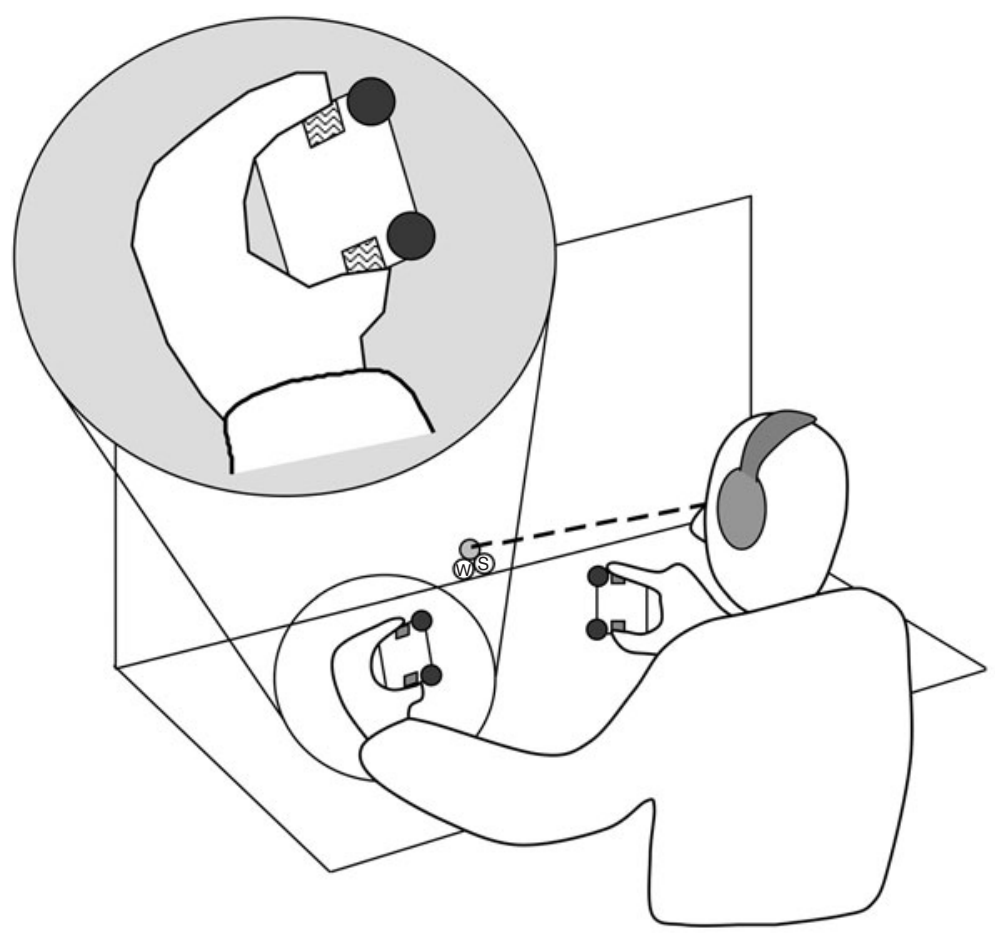

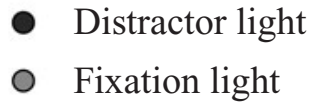

图 Vibrotactile stimulator

(S) (1) Feedback lights

Figure 1. Schematic view of the apparatus and participant in Experiment 1. The participant held a foam cube in each hand. Two vibrotactile stimulators and two visual distractor lights (zig-zag shaded rectangles and filled circles, respectively, in the enlarged inset) were embedded in each foam block, positioned next to the participant's thumb or index finger. White noise was presented continuously over headphones to mask the sound of the operation of the vibrotactile stimulators and footpedals. All of the participants wore an eye movement monitor to measure the direction of their fixation (dotted line). The participants made speeded elevation discrimination responses (by raising the toes or heel of the right foot) in response to vibrotactile targets presented either from the "top" by the index finger of one or the other hand or from the "bottom" by one or the other thumb, respectively.

presented from the top and the visual distractor from the bottom or vice versa) than when they are presented from the same congruent elevation (i.e., either both up or both down). The difference in performance between incongruent and congruent trials for distractor lights provides a measure of the cross-modal congruency effect for a given region of space (e.g., see Maravita et al., 2003), which can index the extent to which a visual distractor intrudes into a vibrotactile judgment.

Several studies have now reported that cross-modal congruency effects can be more pronounced when the vibrotactile target and the visual distractor stimuli are presented from approximately the same location (e.g., from the same foam block) than when they come from different locations (i.e., from two different foam blocks, one held by one hand and the other by the other hand; see Maravita et al., 2003, for a recent review). Cross-modal congruency effects appear to be largest when the visual distractor is presented in the peripersonal space surrounding the hand that receives the target vibration and might, perhaps, fall off as the visual distractor is moved away from the hand into extrapersonal space (see Làdavas, 2002, for a review of potentially related findings from neuropsychological studies of brain-damaged patients suffering from a deficit known as cross-modal extinction; see also the General Discussion section).

Some researchers have already used the cross-modal congruency task in normals to investigate possible changes in the representation of peripersonal space (and in the representation of the body schema), that may be elicited by the prolonged use of hand-held tools (see Holmes, Calvert, \& Spence, 2004; Holmes \& Spence, 2004; Maravita, 
Spence, Kennett, \& Driver, 2002) or by the viewing of realistic rubber hands (Pavani, Spence, \& Driver, 2000) or less realistic artificial body parts (see Austen, Soto-Faraco, Pinel, \& Kingstone, 2001), when they are placed close to the participant's own body. Spatial variations in the magnitude of cross-modal congruency effects from visual distractors on tactile judgments have also been used to investigate the possible nature of representations of peripersonal space when we can see our own limbs only indirectly, via mirror reflections (Maravita, Spence, Sergent, \& Driver, 2002) and even to investigate whether the shadows cast by our own body parts can also, in some sense, become incorporated into the body schema (Pavani $\&$ Castiello, 2004). Finally, the cross-modal congruency task has also now been used to investigate any deficits in the multisensory representation of peripersonal space in brain-damaged patients, such as split-brain patients who have had their cerebral commissure sectioned in order to ameliorate the symptoms of intractable epilepsy (e.g., see Spence, Kingstone, Shore, \& Gazzaniga, 2001; Spence, Shore, Gazzaniga, Soto-Faraco, \& Kingstone, 2001).

Despite the increasingly widespread use of the crossmodal congruency task to investigate various complex questions related to the multisensory representation (and modification) of peripersonal space and/or body schema (see Maravita et al., 2003, for a review), many basic but important empirical questions still remain unanswered. For instance, it is at present unknown whether cross-modal congruency effects are influenced by the direction of endogenous tactile spatial attention to one particular hand or the other or by the relative time of onset of the target and the distractor stimuli. We also do not know the extent to which cross-modal congruency effects are spatially modulated by the relative position of a tactile target, of any distractors, and of the participants' hands within one or the other hemifield in external space, nor do we even know whether cross-modal congruency effects follow the hands very closely when they shift through space, either within a hemifield or when they are crossed over the midline. Providing answers to such questions should allow for a more detailed comparison of the representation of visuotactile space in normal human participants with that inferred from single-cell studies in monkeys (e.g., see Graziano \& Botvinick, 2002, for a review), with the published literature on patients suffering from cross-modal extinction (see, e.g., Làdavas, 2002, for a review), as well as with the results of other studies of normal participants in which different behavioral tasks and/or performance measures have been used to study cross-modal space (see Driver \& Spence, 2004, and Spence, McDonald, \& Driver, 2004, for reviews). In Experiment 1, we showed, rather surprisingly, that directing tactile attention endogenously to one or the other hand has no significant effect on the spatial distribution or magnitude of the cross-modal congruency effects observed. The magnitude of the cross-modal congruency effect is, however, shown to be modulated by the stimulus onset asynchrony (SOA) between the target and the distractor stimuli. In Experiment 2, we provided the first empirical demonstration that the magnitude of the cross-modal congruency effect is reduced as the spatial separation between the tactile target and the visual distractor stimuli increases, even when both the target and the distractor are presented from within the same hemifield. Finally, in Experiment 3, we showed that the representation of visuotactile space is updated when the hands are crossed over the midline, in accord with the available neurophysiological and neuropsychological data on related issues (see Maravita et al., 2003).

\section{EXPERIMENT 1}

We first investigated whether cross-modal congruency effects are affected by the degree of uncertainty regarding the likely location of the vibrotactile target. In particular, we assessed whether the magnitude of any cross-modal congruency effects from visual distractors on tactile judgments would be reduced if participants could focus their tactile endogenous spatial attention on a particular side/ hand in advance. In the experimental situations that have typically been used in previous studies, participants were always uncertain as to which side/hand would receive the target on a particular trial (see, e.g., Holmes, Calvert, \& Spence, 2004; Maravita, Spence, Kennett, \& Driver, 2002; Maravita, Spence, Sergent, \& Driver, 2002; Pavani et al., 2000) and so, presumably, initially had to divide their attention equally between their two hands and, thus, between all the lateral locations at which visual distractors might appear.

Previous research has revealed that a person's ability to determine the elevation of a vibrotactile target presented unpredictably to the thumb or the index finger of either hand (in the absence of any distractor stimulus) can be modulated by the distribution of endogenous tactile spatial attention. For instance, Spence, Pavani, and Driver (2000) reported that people could discriminate the elevation of vibrotactile targets more rapidly and accurately when attending in advance to the target hand/side than when their attention was misdirected toward the other hand/side. Their results thus demonstrated that performance on the vibrotactile elevation discrimination task can be sensitive to the endogenous distribution of tactile spatial attention (see Driver \& Spence, 2004, for a review). Moreover, Kennett, Spence, and Driver (2002) have shown that the peripheral presentation of a spatially nonpredictive visual cue close to either hand (consisting of the illumination of the upper and lower lights together on one or the other of the foam blocks held in the participant's hands) can also lead to a short-lasting facilitation of vibrotactile elevation discrimination responses for a target presented on the cued side, presumably attributable to a reflexive shift of exogenous covert cross-modal spatial attention to the visually cued hand (see Spence, 2001, and Spence et al., 2004, for reviews).

Given such previous results, we considered it likely that the participants in the present study should also find it easier to discriminate the elevation of vibrotactile targets 
when the location (i.e., hand) to which the vibrotactile target was to be presented was made predictable throughout a whole block of trials than when the target hand was kept uncertain throughout a block of trials. The new question was whether this would make it easier to ignore visual distractors, thus leading to reduced cross-modal congruency effects. The ability to respond selectively to vibrotactile information and to ignore irrelevant visual information might be enhanced if participants knew in advance which hand the vibrotactile target would be presented to, so that they could direct their endogenous tactile spatial attention accordingly. But this has, in fact, never been put to experimental test. In order to address this question empirically, the side/hand of the tactile target was made certain in half of the blocks of trials in Experiment 1, whereas it remained uncertain in the remainder of the blocks (only the latter situation had been used in previous studies of the cross-modal congruency task in normal participants; see, e.g., Austen et al., 2001; Holmes, Calvert, \& Spence, 2004; Maravita, Spence, Kennett, \& Driver, 2002; Maravita, Spence, Sergent, \& Driver, 2002; Pavani \& Castiello, 2004; Pavani et al., 2000). As will be seen, our new results were surprising, with the cross-modal congruency effects being found to be entirely unaffected by the direction of endogenous tactile attention, even though this factor did affect tactile performance.

Another aim of our first experiment was to investigate whether the magnitude of the cross-modal congruency effect would be affected by variations in the SOA between the vibrotactile target and the visual distractor stimuli (see C. W. Eriksen \& Hoffman, 1972, for a similar investigation of the time course of intramodal, purely visual congruency effects, also known as flanker interference effects). To this end, the visual distractors in Experiment 1 were unpredictably presented either shortly (i.e., $30 \mathrm{msec}$ ) before the vibrotactile targets, at the same time as the vibrotactile targets, or shortly $(30 \mathrm{msec})$ after the vibrotactile targets. On the basis of unimodal research (e.g., C. W. Eriksen \& Hoffman, 1972), we predicted that the participants would find it harder to ignore the visual distractors when they were presented slightly earlier in time relative to the onset of the vibrotactile targets. However, a rival prediction might be that strictly simultaneous visual and tactile stimuli should interfere the most (cf. Bolognini, Frassinetti, \& Làdavas, 2003; Frassinetti, Bolognini, \& Làdavas, 2002). Our first experiment should help to provide guidelines for maximizing the size of cross-modal congruency effects from visual distractors on tactile judgments, which should prove useful in future research when more subtle questions relating to the multisensory representation of peripersonal space are investigated, as in our subsequent experiments.

\section{Method}

Participants. Twelve participants ( 5 women and 7 men) with a mean age of 27 years (ranging from 20 to 35 years) were recruited through advertisement. All were naive regarding the purpose of the study. All were right-handed by self-report and reported having normal touch, plus normal or corrected vision. The experimental session lasted approximately $45 \mathrm{~min}$. All of the experiments reported here were conducted in accordance with the ethical standards laid down in the 1964 Declaration of Helsinki, and the participants gave their informed consent to participate in the study prior to the start of the experimental session.

Apparatus and Materials. The participant sat at a table in a dimly illuminated room, with head movements minimized by use of an adjustable chinrest. A yellow LED (luminance, $41 \mathrm{~cd} / \mathrm{m}^{2}$ ), placed $60 \mathrm{~cm}$ in front of the participant, at an angle of approximately $25^{\circ}$ below eye level, served as the central fixation point. Two green LEDs (also with a luminance of $41 \mathrm{~cd} / \mathrm{m}^{2}$ ) were placed directly below the fixation light to provide feedback regarding the correctness of each response in the tactile task. The participant rested his/her forearms on a table and held a foam block (length, $60 \mathrm{~mm}$; height, $80 \mathrm{~mm}$; depth, $60 \mathrm{~mm}$ ) between the forefinger and the thumb of either hand (see Figure 1). We used foam blocks because of their ability to dampen any vibrations from the vibrotactile target stimuli, so that they were felt at just the directly stimulated finger or thumb.

Four Oticon-A $(100 \Omega)$ bone conduction vibrators (Oticon Ltd., Somerset, New Jersey; p/n: BC461-1 100), with vibrating surfaces $1.6 \mathrm{~cm}$ in width and $2.4 \mathrm{~cm}$ in length, were used to present each vibrotactile target. The vibrators were attached to the foam blocks and were placed directly under the thumb and forefinger pads of each hand (see the inset in Figure 1). Each sponge block was located so that the vibrators were at a lateral eccentricity of $27^{\circ}$ from central fixation. The vibrators were driven by a $200-\mathrm{Hz}$ sine wave signal fed through an audio amplifier (Eagle, PA2000), with rise and fall times of approximately $20 \mathrm{msec}$ (see Bradshaw, Howard, Pierson, Phillips, $\&$ Bradshaw, 1992). When activated, these vibrotactile stimulators gave rise to a clearly perceptible and easily localizable suprathreshold vibrotactile sensation. Four red LEDs (each with a luminance of $64 \mathrm{~cd} / \mathrm{m}^{2}$ ) were also mounted on the foam blocks (two on each) to provide the visual distractor stimuli, one placed directly next to each of the four vibrotactile stimulators (see Figure 1). Each target stimulus consisted of three 50 -msec bursts of vibrotactile stimulation, all delivered through one or another of the four vibrators, with each burst separated by a $50-\mathrm{msec}$ empty interval. Each visual distractor stimulus also consisted of three pulses of 50-msec duration, separated by $50 \mathrm{msec}$, delivered from any one of the four distractor LEDs. There was a single vibrotactile target consisting of three pulses and a single visual distractor, also comprising three pulses, on each trial.

The participants made speeded discrimination responses regarding the elevation from which the vibrotactile targets were presented (i.e., whether they came from one of the upper locations at a forefinger or from one of the lower locations at a thumb), regardless of the side (left or right hand) from which they were presented. This task required an elevation discrimination response of approximately $10^{\mathbf{}}$ vertically in external space, equivalent to a finger/thumb discrimination in purely somatotopic space. The participants responded via two foot pedals (to keep their hands free for receiving the vibrotactile targets) that were depressed throughout each block of trials. One pedal was situated below the toes, and the other below the heel of the participant's right foot. The participants raised their toes briefly to indicate an upper target or raised their heels to indicate a lower target. White noise was presented to the participants over headphones at $70 \mathrm{~dB}(\mathrm{~A})$ throughout all of the experimental blocks, to mask any sounds made by the operation of the vibrators or the foot pedals.

The timing of the stimuli and responses was controlled by a computer (IBM 386 compatible), using a custom program written in Turbo Pascal 6.0. Response latencies were measured in milliseconds from target onset, using an 82C54 interval timer chip on a digital inputoutput card (DCM-16, Blue Chip Technology, Deeside, Scotland) that was interfaced to the fixation, feedback, and distractor LEDs, plus the vibrotactile stimulators and the foot pedals. The horizontal position of the left eye was monitored for all the participants by means of a Skalar infrared eye tracker (Model 6500, Delft, The Netherlands; see the Procedure section for details). This eye moni- 
tor was connected to the microcomputer controlling the experiment via an analog-to-digital interface board (ADC-42, Blue Chip Technology, Deeside, Scotland).

Design. The four within-subjects factors were the congruency of the visual distractor with respect to the elevation of the vibrotactile target (congruent vs. incongruent in elevation), the visual distractor side with respect to the vibrotactile target side (same vs. different), the predictability of the vibrotactile target side (predictable throughout a block vs. unpredictable on each trial in a block of randomly intermingled left-hand and right-hand targets), and the SOA between the onsets of the target and the distractor stimuli (three levels: visual distractor led by $30 \mathrm{msec}$, simultaneous presentation of target and distractor, or vibrotactile target led by $30 \mathrm{msec}$ ).

There were three blocks of 15 practice trials each, which were not analyzed. In the first such practice block, vibrotactile targets (but no visual distractors) were presented in order to accustom the participants to the vibrotactile elevation discrimination task. Visual distractors were then introduced in the subsequent two practice blocks. The three practice blocks were followed by eight experimental blocks of 64 trials each. These blocks alternated between the vibrotactile targets being presented predictably on only one side (either the left or the right hand; there were predictable target-side blocks for touch, but note that the side, as well as the elevation, of the visual distractor remained unpredictable throughout) and the vibrotactile targets being presented unpredictably on either the left or the right hand in a random sequence (unpredictable target-side blocks). Half of the participants started with a predictable target-side block, whereas the remainder began with an unpredictable target-side block. They were instructed verbally as to the predictable or unpredictable target side for the upcoming block.

In the predictable target-side blocks, equal numbers of vibrotactile targets were presented from each of the two possible target positions (up vs. down) on just one of the foam blocks (i.e., the one held in either the left or the right hand), with target elevation still unpredictable. In the unpredictable target-side blocks, equal numbers of vibrotactile targets were presented from either foam block at either elevation. Visual distractors were always presented randomly, but with equal frequency, from any one of the four possible distractor locations in both the unpredictable and the predictable target-side blocks. The SOA was selected randomly on each trial.

Procedure. At the beginning of the experiment, the participants were instructed to make speeded elevation discrimination responses to the vibrotactile target stimuli. They were also informed that visual distractors would be presented together with the vibrotactile targets but that they were completely irrelevant to their vibrotactile elevation discrimination task. Indeed, it was made explicit to the participants that they should try to ignore the visual distractors as much as possible (while keeping their eyes open throughout, as confirmed with the eye tracker). The participants were also instructed to maintain their fixation on the central fixation light that was illuminated at the start of each trial until it was extinguished at the end of the trial. The first stimulus from the pair of vibrotactile target and visual distractor stimuli on each trial (which modality led depended on the SOA) was presented $900-1,050 \mathrm{msec}$ after the onset of the fixation light at the start of the trial.

At the beginning of each block of trials, the participants were informed about the expected side(s) for the vibrotactile targets (either, predictably, just the left or the right hand or, unpredictably, either side, depending on the block type). For blocks of trials in which the vibrotactile targets predictably appeared on only one side, the participants were instructed to direct their tactile spatial attention to that side (cf. Spence, 2002), while always maintaining central fixation. For blocks of trials in which the vibrotactile stimuli were equally likely to appear on either side, the participants were instructed to divide their tactile attention equally between the two possible target sides in advance. They were instructed to respond as rapidly as possible, while making as few errors as possible. Note that the partici- pants always adopted exactly the same posture, with each uncrossed hand holding a foam block at a fixed location (see Figure 1), no matter which type of block (predictable or unpredictable target side) they were performing.

If no discrimination response was made within 1,500 msec of target onset, the trial was terminated; otherwise, the participant's response ended the trial. Visual feedback was presented whenever an erroneous response was made, by illuminating one of the two green LEDs. One LED, inscribed with the letter "S" (for slow response), was illuminated on trials in which no response had been made within $1,500 \mathrm{msec}$ of target onset, and the other, inscribed with the letter "W" (for wrong response), was illuminated on trials in which the participant raised the wrong foot pedal. These feedback lights were flashed for $960 \mathrm{msec}$ on trials in which the participants made an incorrect or slow response. Any feedback was followed by a further delay of $400 \mathrm{msec}$ before the onset of the next trial. After the response and any feedback, there was a further delay of $1,000 \mathrm{msec}$ before the fixation light came on again to signal the start of the next trial.

Eye movements were monitored for all the participants throughout the experiment, to ensure that any differences between performance in the predictable and the unpredictable target side blocks could not be attributed simply to the participants' selectively looking toward the stimulated target hand just during the predictable targetside blocks (which could have trivially modulated visual processing for the distractors on one side vs. the other). The eye movement monitor was calibrated to provide a signal to the computer whenever an eye movement of $2^{\circ}$ or more was detected in the period between the onset of the cue and the initiation of a choice discrimination response by the participant. This was the smallest signal that could be consistently detected across participants with the apparatus used, but note that the peripheral targets were located very far from central fixation (i.e., at an eccentricity of $27^{\circ}$ ). The monitor was recalibrated to confirm straight-ahead at the beginning of every block of trials, and also during a block, if fixation appeared to drift from the calibrated center point. Trials on which potential eye movements were detected (note that blinks sometimes resulted in signals that were indistinguishable from actual eye movement signals) were automatically excluded from the subsequent analysis of the data.

\section{Results}

Trials with an incorrect response were discarded from the reaction time (RT) analysis. Due to excessive eye movements (or blink artifacts), 9.6\% of the trials were removed, and $0.8 \%$ of the trials were removed across all participants because no response was made within $1,500 \mathrm{msec}$ of the target onset. The interparticipant mean RTs (after these exclusions), together with the corresponding error rates for all trials and the mean cross-modal congruency effects (derived by subtracting incongruent from congruent conditions), are shown in Table 1 as a function of target-side predictability, relative distractor side, target-distractor congruency, and SOA. The major results are also summarized in Figure 2.

The data were analyzed using a within-subjects analysis of variance (ANOVA) with four factors: target-distractor congruency $(2) \times$ relative distractor side $(2) \times$ target-side predictability (2) $\times$ SOA (3). RT analysis revealed a significant main effect of target-distractor congruency $[F(1,9)=31.1, p<.0003]$, caused by the participants' responding more rapidly on congruent distractor trials $(M=475 \mathrm{msec})$ than on incongruent distractor trials $(M=$ $534 \mathrm{msec}$ ) overall, as was expected. These cross-modal congruency effects were larger when the visual distractor 
Table 1

Mean Reaction Times (RTs, in Milliseconds), Their Standard Deviations, Percentages of Errors (\%E), and Mean Cross-Modal Congruency Effects (CCEs, Calculated as Incongruent Minus Congruent Conditions) for Vibrotactile Targets in Experiment 1, as a Function of Relative Distractor Side, Target-Distractor Stimulus Onset Asynchrony (SOA), Target-Distractor Congruency, and Target Side Predictability

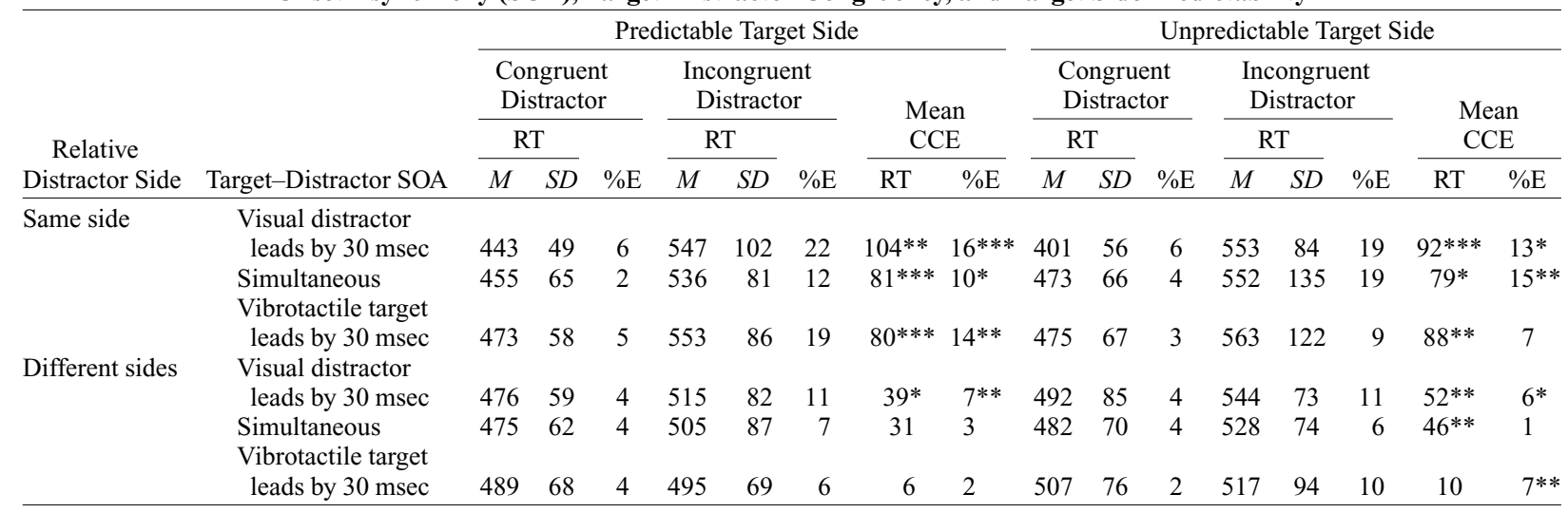

${ }^{*} p<.05 . \quad * * p<.01 . \quad * * * p<.001$.

was presented from the same side/hand as the vibrotactile target (mean incongruent minus congruent difference of $87 \mathrm{msec}, p<.0002$ ) than when the visual distractor appeared from the opposite side/hand (mean cross-modal congruency effect reduced to only $30 \mathrm{msec}, p<.01$ ), resulting in a significant interaction between target-distractor congruency and relative distractor side $[F(1,9)=17.5$, $p<.003$; see Figure 2A].

Cross-modal congruency effects were also modulated by the SOA between the vibrotactile target and the visual distractor, as was revealed by the interaction between target-distractor congruency and SOA $[F(2,18)=3.5$, $p<.05]$. When the onset of the visual distractor occurred $30 \mathrm{msec}$ before that of the vibrotactile target, the mean cross-modal congruency effect was $72 \mathrm{msec}$, as compared with $59 \mathrm{msec}$ when the two stimuli were presented simultaneously and $46 \mathrm{msec}$ when the vibrotactile target preceded the visual distractor (see Figure 2B; the difference between the two asynchronous onset conditions was significant in a two-tailed paired samples $t$ test, $p<.02$ ).

The RT analysis also revealed a significant main effect of target-side predictability $[F(1,9)=7.8, p<.02]$, reflecting the fact that tactile judgments were more rapid overall in the predictable target-side blocks $(M=497 \mathrm{msec})$ than in the unpredictable target-side blocks $(M=512 \mathrm{msec})$, as would be expected. However, there was no interaction between target-side predictability and target-distractor congruency or between target-side predictability, targetdistractor congruency, and relative distractor side (all $F_{\mathrm{S}}<1$ ). This suggests that, contrary to the expectations outlined in the introduction, the magnitude of the crossmodal congruency effects reported in Experiment 1 was quite unaffected by the distribution of endogenous, or voluntary, spatial attention (i.e., whether endogenous spatial attention was focused on just one side/hand or was divided equally between the two sides/hands; see Figure 2C), even though overall responding was faster with attention focused on the expected side for the tactile target. None of the other terms in the RT analysis reached significance (all $F \mathrm{~s}<2$ ).

A similar analysis of the error data also revealed a significant main effect of target-distractor congruency $[F(1,9)=24.5, p<.0008]$, caused by the participants making more errors on incongruent trials $(M=12.5 \%)$ than on congruent distractor trials $(M=3.9 \%)$ overall, consistent with the RT congruency effect. There was also a significant main effect of relative distractor side $[F(1,9)=$ $44.0, p<.0001]$, reflecting the fact that the participants made more errors when the vibrotactile target and the visual distractor stimuli appeared on the same side/hand $(M=10.4 \%)$ than when they were presented from different sides/hands $(M=6.0 \%)$. In fact, the participants showed a larger cross-modal congruency effect in errors when the target and the distractor stimuli were presented to the same side/hand (mean effect of $12.5 \%$ ) than when they were presented from opposite sides/hands (mean effect of $4.6 \%$ ), resulting in an interaction between target-distractor congruency and relative distractor side $[F(1,9)=30.9, p<$ .0004], as was also found for RTs.

The participants also made more errors when the onset of the visual distractors occurred before that of the vibrotactile targets $(M=10.2 \%)$ than when the stimuli were presented simultaneously $(M=7.1 \%, p<.01$, by a paired $t$ test comparison) or when the vibrotactile targets were presented slightly before the visual distractors $(M=7.3 \%$, $p<.02$, by paired $t$ test comparison), leading to a significant main effect of SOA $[F(2,18)=6.6, p<.007$; see Figure 2B). Finally, the interaction between SOA, targetside predictability, and relative distractor side also reached significance $[F(2,18)=5.3, p<.02]$. However, visual inspection of Table 1 does not provide any theoretically motivated explanation for this unexpected term, and so it will 

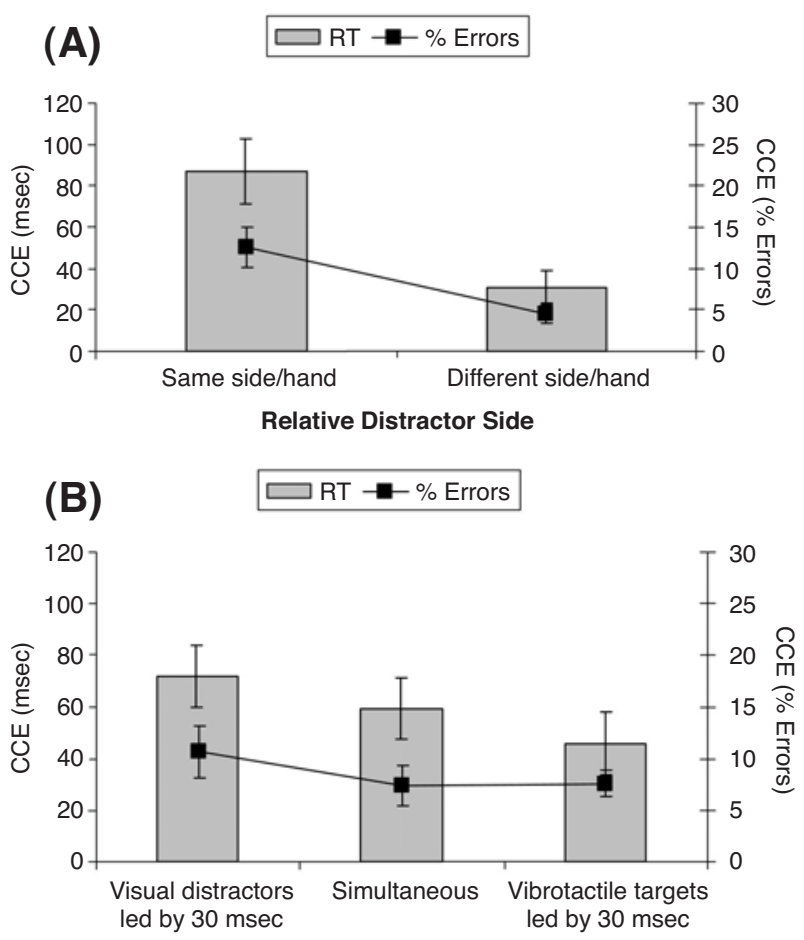

Target-Distractor SOA

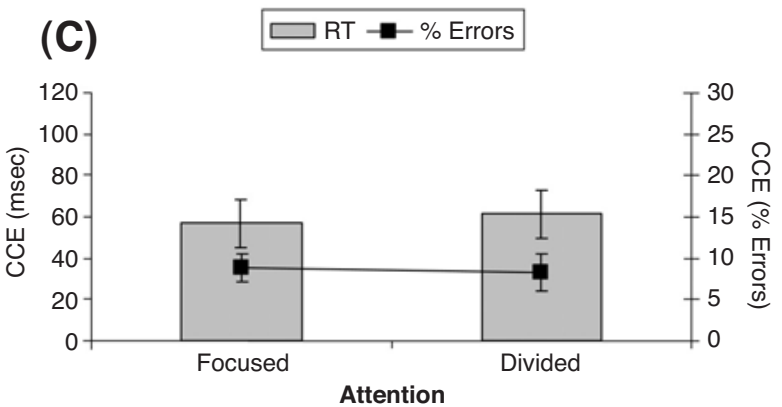

Figure 2. Graphs showing modulation of the cross-modal congruency effect (CCE) as a function of spatial, temporal, and attentional manipulations introduced in Experiment 1. In all three panels, the filled bars show the cross-modal congruency effects in the reaction time (RT) data, whereas the line plots show the crossmodal congruency effects in terms of the error data. The error bars indicate the standard error of the means. (A) The cross-modal congruency effects were greater when the vibrotactile target and the visual distractor stimuli were presented from the same foam block (i.e., from the same lateral spatial location or the same side) and were smaller when the target and the distractor stimuli came from different sides (the azimuthal distance between the target and the distractor in the different-sides condition was $63^{\circ}$ laterally). (B) Cross-modal congruency effects were larger when the onset of the visual distractors preceded the onset of the vibrotactile targets by $30 \mathrm{msec}$, with the effect declining if the onsets of the two stimuli occurred simultaneously or if the onset of vibrotactile targets occurred 30 msec before that of the visual distractors. (C) The magnitude of the cross-modal congruency effect was unaffected by whether the participant knew in advance to which hand the vibrotactile target would be presented (focused attention), versus when the targets were presented unpredictably to either hand on each trial (divided attention). not be considered further here. None of the other terms in the analysis of the error data reached statistical significance (all $F_{\mathrm{S}}<2.5$ ).

\section{Discussion}

The results of Experiment 1 clearly demonstrate that the participants could not entirely ignore the irrelevant visual distractor stimuli, despite explicit instructions to do so, when making tactile elevation discriminations (thumb vs. index finger) for vibrotactile targets presented to either of their hands. Significant cross-modal congruency effects were observed overall, and also within the majority of subconditions, as was revealed by slower elevation discrimination responses and increased error rates when the elevation of the visual distractor was incongruent with that of the vibrotactile target, as compared with when it was congruent.

Cross-modal congruency effects were greatest when the vibrotactile target and the visual distractor were presented at the same side/hand (i.e., from the same foam block) and were significantly reduced when the target and the distractor were presented from different sides/hands (i.e., from different foam blocks; see Figure 2A). Given that the present findings were obtained under conditions in which we were able to ensure that the participants successfully maintained central fixation (by explicitly monitoring eye position), these results provide some of the most convincing evidence to date that the spatial modulation of crossmodal congruency effects cannot be explained simply in terms of overt orienting, such as if the participants had a tendency to look toward the visual distractors near the stimulated hand (see Driver \& Grossenbacher, 1996, for the potential importance of gaze direction in even purely tactile tasks). Moreover, this spatial modulation of the cross-modal congruency effect occurred here despite the fact that the visual distractors were presented from within peripersonal space in all the conditions. Therefore, our results could be taken to suggest that the magnitude of the cross-modal congruency effect may be modulated by the spatial distance between the target and the distractor stimuli in external space, rather than simply being a function of whether the visual distractor is presented within peripersonal space, near the body overall, rather than in more distant extrapersonal space (cf. Làdavas, 2002; Rizzolatti et al., 1997).

Two further important new findings also emerged from Experiment 1. A surprising result was that while making the target side/hand entirely predictable facilitated response latencies overall (thus showing that our participants did direct covert spatial attention endogenously toward the expected target hand), the magnitude of the crossmodal congruency effect was not affected by such focusing of attention on one hand (see Figure 2C). These results therefore suggest that the spatial modulation of the crossmodal congruency effect is rather immune to the endogenous distribution of spatial attention. That is, our participants were apparently no more able to filter out irrelevant visual distractors, to prevent these from influencing the 
efficiency of their tactile judgments, when the target side/hand was entirely predictable for the tactile task than when it was unpredictable.

The improvement in overall performance for the tactile task when the target hand was known agrees with previous work on endogenous spatial attention in tactile tasks (e.g., Lloyd, Merat, McGlone, \& Spence, 2003; Soto-Faraco, Ronald, \& Spence, in press; Spence, Ranson, \& Driver, 2000; see also Spence, Shore, \& Klein, 2001). But no visual distractor stimuli had been presented in any of these previous studies. Here, we find for the first time that the improved tactile performance on an endogenously attended hand does not reduce cross-modal congruency effects from visual distractors. It might be that when participants direct their endogenous spatial attention to one side/hand or the other, this not only facilitates their perception of the vibrotactile target stimuli, but also (unwittingly) enhances their perception of the visual distractor stimuli presented from the same side/hand, thus entailing that visual distractor effects are maintained. Previous research has indeed shown that when people try to direct their tactile spatial attention to a particular side, their visual attention will often typically follow to the same hand/spatial location (see Spence, Pavani, \& Driver, 2000, Experiment 6; see also Driver \& Spence, 2004; Eimer, 2004; Eimer \& Driver, 2000). The existence of such crossmodal links in endogenous spatial attention might explain why no reduction of the cross-modal congruency effect (or any significant change in the spatial modulation of the congruency effect) was found in the predictable (as compared with the unpredictable) target- side blocks of Experiment 1 .

The second new finding to emerge from Experiment 1 was that small changes in the SOA between the onset of the vibrotactile target and the onset of the visual distractor stimuli resulted in a significant modulation of the overall cross-modal congruency effects observed (see Figure 2B). In particular, larger cross-modal congruency effects were observed in the RT data when the onset of the visual distractors preceded that of the vibrotactile targets (by $30 \mathrm{msec}$ ) than when their onset occurred shortly after that of the vibrotactile targets (intermediate results were reported when the target and the distractor were presented simultaneously). This temporal modulation of the present cross-modal congruency effects matches similar results reported previously in purely visual (i.e., intramodal) congruency studies, such as those in which the Eriksen flanker task was used (e.g., C. W. Eriksen \& Hoffman, 1972). In the remainder of this study, we used just the SOA that produced the biggest cross-modal congruency effect of the three SOAs we had tested (i.e., when the visual distractors led by $30 \mathrm{msec}$ ), to maximize the cross-modal congruency effect observed when using it as a measure for studying spatial issues.

\section{Are Cross-Modal Congruency Effects Modulated by Target-Distractor Spatial Separation?}

In Experiment 1, the vibrotactile target and visual distractor stimuli that were presented on the same side/hand projected to the same cerebral hemisphere. By contrast, when the target and the distractor stimuli are presented from different sides/hands, they will project, at least initially, to different cerebral hemispheres. Consequently, there are at least two possible (and by no means necessarily exclusive) explanations for the apparently "spatial" modulation of the cross-modal congruency effect reported in Experiment 1 (i.e., for the same- vs. different-side/hand difference in cross-modal congruency effects; see Figure 2A). First, it may be that the magnitude of the crossmodal congruency effect depends on the separation in external space between the vibrotactile target and the visual distractor. According to this "spatial" account, simply presenting the target and the distractor stimuli from different spatial positions within the same hemifield (and thus without necessarily changing the cerebral hemisphere to which the two stimuli project initially) should result in a decrease in the magnitude of the cross-modal congruency effect, when compared with the situation in which the target and the distractor are presented from exactly the same external position (i.e., from the same foam block).

Alternatively, it could be argued that the larger crossmodal congruency effects reported in Experiment 1 when the target and the distractor stimuli were presented to the same side/hand, rather than from different sides/hands, might simply reflect a difficulty inherent in selecting the relevant vibrotactile spatial information (and ignoring the irrelevant distracting visual spatial information) when the stimuli from both sensory modalities project initially to the same cerebral hemisphere (rather than from the same spatial position). According to the latter "hemispheric projection" account, the reduction of the cross-modal congruency effect seen when the target and the distractor stimuli were presented from different sides/hands might simply reflect the fact that the participants found it easier to select between competing sensory inputs when they were processed, at least initially, by different cerebral hemispheres (see Banich, 1998; Friedman \& Polson, 1981; Kinsbourne \& Cook, 1971; Kinsbourne \& Hicks, 1978; Merola \& Liederman, 1985; and Passarotti, Banich, Sood, \& Wang, 2002 , on the topic of hemispheric attentional resources).

Unequivocal evidence in support of either (or both) of these two interpretations cannot be derived from the results of Experiment 1, since the same- versus differentside factor was perfectly confounded with whether the visual and the tactile stimuli presented together on any trial would project initially to the same versus different cerebral hemispheres. Therefore, in Experiment 2, we looked more directly for any evidence to support the spatial account by examining whether varying the proximity between the target and the distractor stimuli in external space (without necessarily changing the hemisphere to which they should initially project) would modulate the magnitude of crossmodal congruency effects. Any such result would provide support for there being at least some "spatial" modulation of cross-modal congruency effects (we further assessed the evidence for any hemispheric influence on cross-modal congruency effects in Experiment 3). 


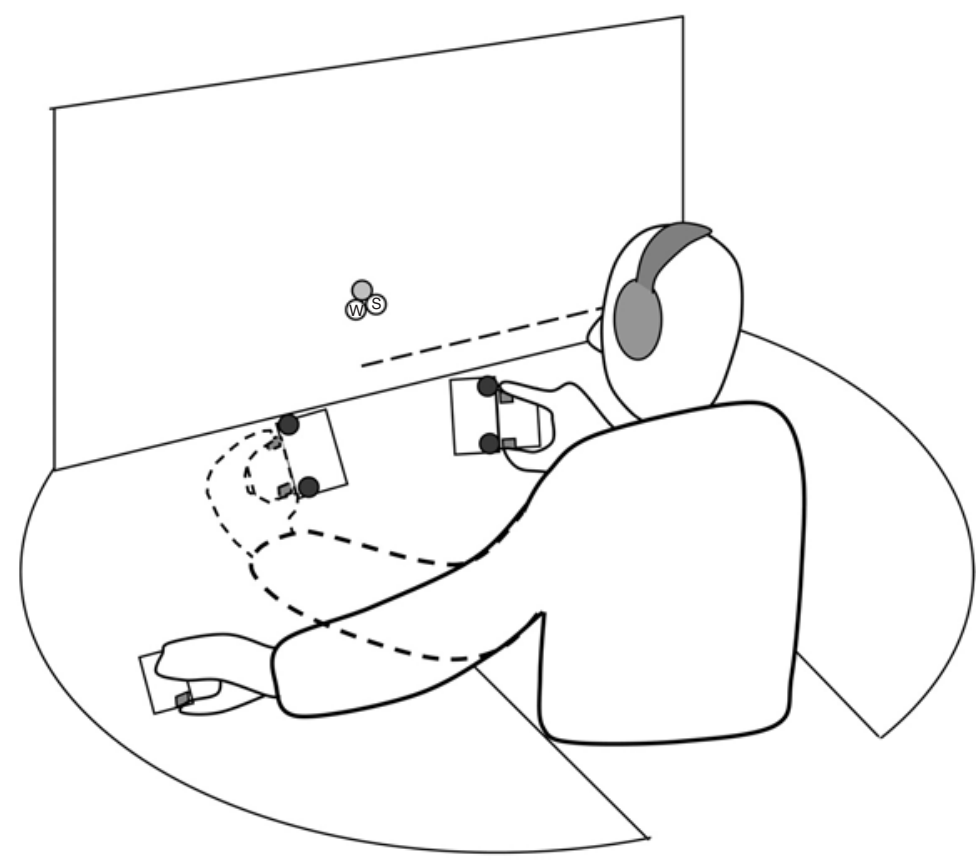

- Distractor light

○ Fixation light
Vibrotactile stimulator

(S) (10) Feedback lights

Figure 3. Schematic view of the apparatus and the two possible postures adopted by the participants in Experiment 2. The boxes on which the visual distractor light-emitting diodes were mounted are shown attached to the tabletop in front of the participant. The foam blocks in which the vibrotactile stimulators were mounted could be inserted into the more eccentric sides of these boxes (as is shown for the foam block held in the right hand in the figure). The handsnear posture is shown by the dashed outline of the left hand and arm, whereas the hands-far posture is represented by the solid outline of the left hand. Note that for half of the participants, the left arm was the mobile arm (as is shown here), whereas for the remainder, the right arm was the mobile arm (and the left hand adopted a stationary posture by the left distractor lights). The mobile hand was held stationary within blocks, being placed in one or the other posture between blocks. Eye position was once again monitored by an eye movement monitor (fixation is shown by the dotted line), while white noise was presented continuously throughout each block of trials over headphones.

\section{EXPERIMENT 2}

Participants made speeded elevation discrimination responses to vibrotactile targets presented to the index finger or the thumb of either hand. However, the distracting lights were now mounted on two boxes attached to the top of the table at which the participant sat, instead of being placed directly beside the vibrators on the foam blocks (see Figure 3 for a schematic view of the modified experimental setup used in Experiment 2). With this setup, the vibrotactile stimulators could either be held adjacent to the visual distractors in the same hemifield or could be positioned far from them within the same hemifield. Therefore, both the vibrotactile target and the visual distractor stimuli could still be presented to the same or to different sides/hemifields, relative to the saggital midline, and they also could still project to the same (or a different) cerebral hemisphere; but critically, the spatial proximity between the two stimuli (tactile target and visual distractor) could now be systematically varied independently of this. If the magnitude of the cross-modal congruency effect depends, at least in part, on the spatial arrangement of the target and the distractor stimuli in external space, any cross-modal congruency effects elicited by the visual distractors should be reduced when the tactile target and the visual distractor stimuli were presented far apart from each other within the same hemifield (as compared with when they were presented from the same position within a hemifield; see the two possible left-hand positions sketched in Figure 3). To simplify the experimental design, the vibrotactile target side was always unpredictable in Experiment 2 (although note that we shall return to the use of a predictable targetside design in Experiment 3), and the visual distractor was now always presented $30 \mathrm{msec}$ before the vibrotactile tar- 
get (since this SOA had been shown to result in the largest cross-modal congruency effects in Experiment 1).

\section{Method}

Participants. Twelve participants ( 8 women and 4 men) took part in this experiment. Their mean age was 23 years, with a range from 20 to 25 years. All the participants reported normal touch and normal or corrected vision. All except 2 were right-handed by self-report. Each participant took part in one experimental session of approximately 60-min duration. Three of the participants had taken part in Experiment 1.

Apparatus and Materials. The apparatus and materials were the same as those in Experiment 1, with the exception that the four red LEDs providing the visual distractor stimuli were now mounted in upper/lower pairs on two boxes, instead of being placed directly beside the vibrators on the foam blocks themselves (see Figure 3). The boxes (length, $100 \mathrm{~mm}$; height, $100 \mathrm{~mm}$; depth, $100 \mathrm{~mm}$ ) were made of black cardboard and were fixed to the tabletop in front of the participant, one to the right and the other to the left of the participant's midline. The more eccentric side of each box was situated $27^{\circ}$ from central fixation. An LED was placed by the upper and lower corners of each box and was directed toward the participant. Note that with this apparatus during central fixation, the LEDs occupied exactly the same position in the visual field as that in Experiment 1 . The more eccentric side of each box was open to allow one of the foam blocks to be partially inserted into the box.

In half of the experimental blocks, both foam blocks were held inside the boxes (condition with hands close to each other). In these blocks, the vibrators on each side were adjacent to the LEDs in the same hemifield (just as in Experiment 1). In the remaining experimental blocks, one hand held a foam block inside the box, while the other hand (illustrated for the left hand in Figure 3) now held its foam block at $90^{\circ}$ from the participant's sagittal midline (hands-far condition). With this experimental set up, the vibrators held in the $90^{\circ}$ laterally displaced hand were positioned at an eccentricity of $63^{\circ}$ with respect to the visual distractors positioned in the same hemifield (see the left side of Figure 3). We refer to the stimulated hand that changed position across blocks as the mobile hand (shown for the left hand in Figure 3, but note that this hand did not move during trials, only shifting from one posture to the other between blocks and then resting at a fixed location throughout a block), whereas the stimulated hand that remained stationary across blocks is termed the fixed hand (right hand in Figure 3, but equally likely to be the left hand in the experiment, as counterbalanced across participants). When the arm was extended to the side of the participant's body, a wooden support $(80 \times 80 \mathrm{~cm})$ was added to the table to support the weight of the participant's elbow and forearm. It is important to note that manipulation of posture for the mobile hand did not change the tactile stimuli to the fingers or thumbs themselves or the visual dis- tractors, since the eyes maintained central fixation; only the relative location in external space of tactile stimuli and visual distractors changed substantially.

Design. The four within-subjects factors were the congruency between the vibrotactile target and the visual distractor elevations (congruent vs. incongruent, as before), the relative distractor hemifield with respect to the vibrotactile target (same vs. different), the posture adopted (hands close vs. hands far), and the stimulated hand (fixed hand vs. mobile hand).

There were three blocks of 15 practice trials, followed by four test blocks of 112 trials each. The successive blocks alternated between the two postures (i.e., hands close vs. hands far). Half of the participants started with each hand near to the distractors within its corresponding hemispace, whereas the remainder began with one hand far from the distracting lights within its hemispace. The mobile hand was the right hand for half of the participants and was the left hand for the remainder of the participants. Equal numbers of vibrotactile targets were presented on either side of fixation from each of the four possible somatotopic target locations (i.e., finger or thumb on either hand). Similarly, equal numbers of visual distractors were presented on either side of fixation from each of the four possible retinal distractor locations, regardless of target side or elevation.

Procedure. The procedure was exactly the same as that in Experiment 1, with the exception that the changes of posture for one mobile hand occurred between experimental blocks. The participants were informed at the beginning of the experiment that the vibrotactile targets were equally likely to appear in any of the four possible somatotopic target locations and that any visual distractor was task irrelevant and so should simply be ignored. As in the previous experiment, the eye position of all the participants was monitored to ensure adherence with the central fixation instructions.

\section{Results}

Trials with an incorrect response were discarded from the RT analysis. Due to the excessive eye movements (or blink artifacts), $6.1 \%$ of the trials were removed, and $0.4 \%$ of the trials were removed across all participants because no response was made within $1,500 \mathrm{msec}$ of target onset. Table 2 gives the interparticipant mean results, including cross-modal congruency effects (incongruent minus congruent scores), for both the RT and the error data, as a function of target-distractor congruency, relative distractor side, posture adopted, and stimulated hand. The key results are also summarized schematically in Figure 4.

A preliminary within-subjects ANOVA with one factor (target-distractor congruency [2]) was conducted on the RT and error data, to evaluate the existence of a cross-modal

Table 2

Mean Reaction Times (RTs, in Milliseconds), Their Standard Deviations, Percentages of Errors (\%E), and Mean Cross-Modal Congruency Effects (CCEs) for Vibrotactile Targets in Experiment 2, as a Function of Relative Distractor Hemifield, Posture, Stimulated Hand, and Target-Distractor Congruency

Stimulated Hand

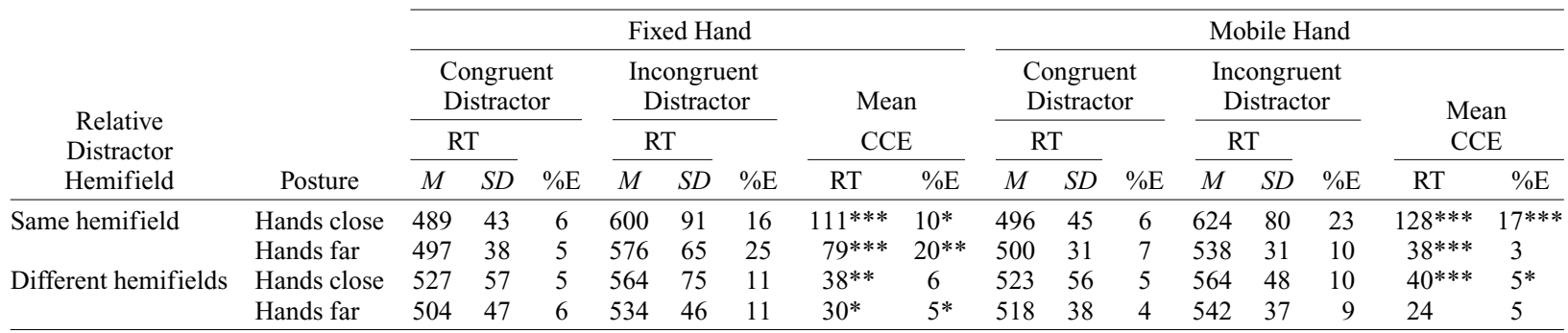

$* p<.05 . \quad * * p<.01 . \quad * * * p<.001$. 
(A)

\author{
Mean CCE $=84 \mathrm{msec}$ \\ $(10.9 \%$ errors $)$
}

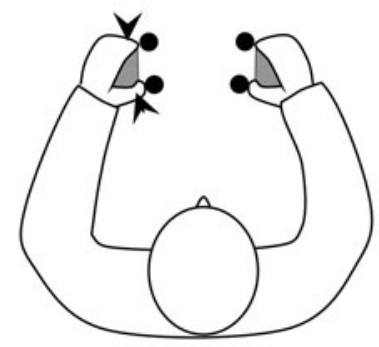

(C) Mean $\mathrm{CCE}=75 \mathrm{msec}$

( $7.8 \%$ errors)

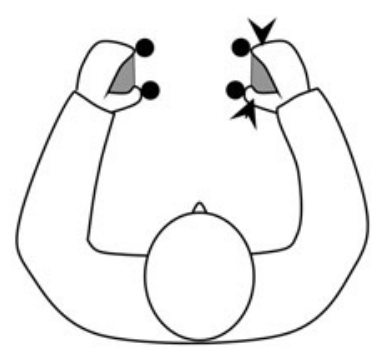

(B)

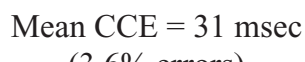

(3.6\% errors)

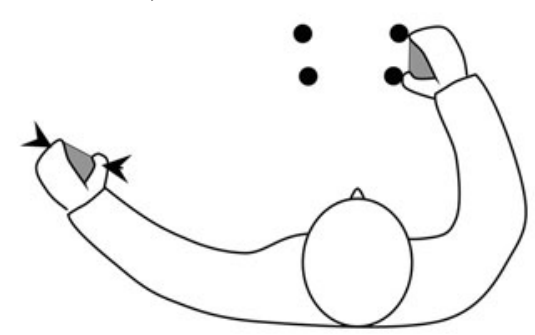

(D) Mean $\mathrm{CCE}=53 \mathrm{msec}$

( $12.4 \%$ errors)

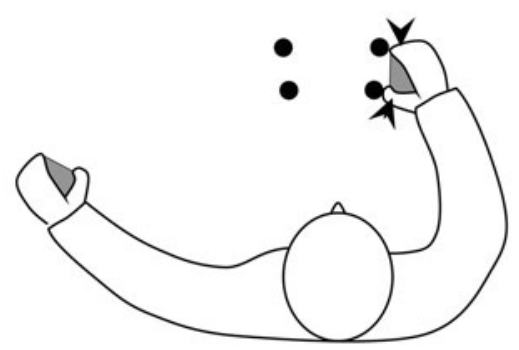

Figure 4. Schematic view of the postures adopted by the participants holding the foam blocks (represented by the shaded and partially occluded rectangles held in the participant's hands) in Experiment 2 and the major results obtained (in particular, the interaction between posture and stimulated hand; see the main text for details). The hands-close posture is shown in panels $A$ and $C$, whereas the hands-far posture is shown in panels $B$ and $D$. The vibrotactile targets (indicated by the filled arrowheads) are shown as presented to the mobile hand in panels $A$ and $B$ and to the stationary hand in panels $C$ and $D$. The position of the distractor lights are shown schematically as filled circles. Mean cross-modal congruency effects (CCEs) elicited by visual distractors are shown numerically (in terms of both the reaction time and the error data) above the foam block on which the relevant vibrotactile targets were presented. The results show clearly that changing the posture of the hand that received the current vibrotactile target had a dramatic effect on CCEs (panels A and B) but that there was very little effect when the stationary hand received the target stimulus instead (panels $C$ and $D$; note also that any simple comparison of just the latter two conditions in terms of either the speed or the accuracy of performance is confounded by the potential speed-accuracy tradeoff present; see the main text and Note 1).

congruency effect in Experiment 2. This analysis revealed that the participants responded more rapidly overall on congruent trials $(M=507 \mathrm{msec})$ than on incongruent trials $[M=568 \mathrm{msec} ; F(1,11)=54.0, p<.0001]$. The participants also made fewer errors on congruent trials $(M=$ $5.3 \%)$ than on incongruent trials $[M=14.0 \% ; F(1,11)=$ $18.8, p<.001]$, replicating the cross-modal congruency effects reported in the previous experiment. Having demonstrated the existence of a basic cross-modal congruency effect, we focused our subsequent analysis on the differences caused by this (i.e., on the cross-modal congruency effects themselves, determined by subtracting congruent values from incongruent values for otherwise matching subconditions), to simplify the interpretation of the statistical data (three-factor rather than four-factor ANOVA). Withinsubjects ANOVAs were thus performed on the cross-modal congruency effects (i.e., difference of incongruent minus congruent conditions for each participant) for the RT and error data, with three factors: relative distractor hemifield (2) $\times$ stimulated hand $(2) \times$ posture $(2)$.

The RT analysis revealed a significant main effect of relative distractor hemifield $[F(1,11)=26.8, p<.0003]$, reflecting the fact that cross-modal congruency effects were again larger when the vibrotactile target and the visual distractor stimuli were presented from the same hemifield $(M=89 \mathrm{msec})$ than when they were presented from different hemifields $(M=33 \mathrm{msec})$. Critically, larger cross-modal congruency effects were also reported in the hands-close posture (with the vibrators on each side being held adjacent to the LEDs positioned in the same hemifield; $M=79 \mathrm{msec}$ ) than in the hands-far posture (with the vibrators on the side being held by the $90^{\circ}$ laterally displaced hand, $63^{\circ}$ from the LEDs positioned in the same hemifield; $M=43 \mathrm{msec}$ ), resulting in a main effect of posture $[F(1,11)=36.6, p<.0001]$. Moreover, this effect of posture emerged only when the vibrotactile targets and 
the visual distractors were presented in the same hemifield (hands close, $M=120 \mathrm{msec}$; hands far, $M=59 \mathrm{msec} ; p<$ $.0005)$, but not when the vibrotactile target and the visual distractor stimuli were presented in different hemifields (39 vs. $27 \mathrm{msec}, \mathrm{n} . \mathrm{s}$.), resulting in an interaction between posture and relative distractor hemifield $[F(1,11)=7.8$, $p<.02]$.

Finally, there was an interaction between posture and stimulated hand $[F(1,11)=6.5, p<.03]$, caused by larger cross-modal congruency effects being reported for vibrotactile targets presented to the mobile hand with hands close ( $M=84 \mathrm{msec}$; see Figure 4A) than when the hands were placed far apart $(M=31 \mathrm{msec}, p<.0008$; see Figure 4B). Note that this effect arises even though posture did not change the tactile targets or the visual distractors themselves but only their relationship in external space. By contrast, the two postures affected the cross-modal congruency effect to a significantly lesser extent when the targets were presented to the fixed hand $(75 \mathrm{vs} .53 \mathrm{msec}$, $p<.05$; see Figures 4C and 4D). It should be noted here that although both of these posture effects on RTs were significant, only the latter effect (for the fixed hand) was actually offset by a slight reverse trend in the error data, raising the possibility of a speed-accuracy tradeoff for just that effect (see below). Overall, these RT results suggest that the magnitude of the cross-modal congruency effect changed according to the posture adopted and did so primarily when the posture affected the distance between the vibrotactile targets and the visual distractors (i.e., for tactile targets presented to the mobile hand). This result provides support for there being some "spatial" component to the modulation of cross-modal congruency effects. None of the other terms in the analysis reached significance (all $F \mathrm{~s}<2.6$ ).

A similar analysis of the mean cross-modal congruency effects present in the error data revealed a significant main effect of relative distractor hemifield $[F(1,11)=16.0, p<$ $.002]$. This was again caused by larger cross-modal congruency effects being reported when the target and the distractor stimuli were presented to the same hemifield $(M=$ $12.5 \%)$ than when they were presented from different hemifields $(M=4.9 \%)$. There was also an interaction between posture and stimulated hand $[F(1,11)=16.4, p<$ $.002]$, reflecting the fact that larger cross-modal congruency effects were reported for vibrotactile targets presented to the mobile hand when the hands were in the hands-close posture $(M=10.9 \%$; see Figure 4A) than when they were in the hands-far posture $(M=3.6 \%, p<$ .01 ; see Figure 4B), in accord with the analogous RT effect. By contrast, no significant effect of posture emerged when the vibrotactile targets were presented to the fixed hand (hands close, $M=7.8 \%$; hands far, $M=12.4 \%, p=$ .13; see Figures $4 \mathrm{C}$ and $4 \mathrm{D}$ ). Note that, as has already been pointed out, the direction of this nonsignificant trend in the error data for the fixed hand was actually the reverse of that seen in the RT data. ${ }^{1}$

Importantly, the more pronounced effect of posture for the mobile hand in the error data was observed only when the vibrotactile target and the visual distractor stimuli were presented to the same hemifield and was absent when they were presented from opposite hemifields [relative distractor hemifield $\times$ stimulated hand $\times$ posture $[F(1,11)=$ 18.3, $p<.001$; see Table 2]. That is, the position of the mobile hand had an effect on the magnitude of crossmodal congruency effects only when it was the hand receiving the vibrotactile target and the visual distractor was presented from within the same hemifield (just as in the analysis of the RT data). None of the other terms in the analysis of the error data reached significance (all $F \mathrm{~s}<1.5$ ).

\section{Discussion}

The results of Experiment 2 replicate the basic visualtactile cross-modal congruency effect reported in Experiment 1 . They also show that when the visual distractor was presented from the same side/hemifield as the vibrotactile target, cross-modal congruency effects were larger than when the target and the distractor stimuli were presented in opposite sides/hemifields. The more important (and novel) result to emerge from Experiment 2 was that when the vibrotactile target was presented to the mobile hand (i.e., the hand that changed its position only between blocks of trials), cross-modal congruency effects from a distractor light in the same hemifield (initially projecting to the same hemisphere as the target) were modulated by the relative position of the hand with respect to the light in external space within that hemifield. Crucially, this result was obtained despite the fact that this manipulation of hand position did not affect the somatotopic representation of the tactile targets or the retinal visual distractors themselves. In particular, larger cross-modal congruency effects were reported when a tactile target on the mobile hand was directly adjacent to a visual distractor in the same hemifield than when the hand was placed away from this (i.e., in the $90^{\circ}$ position, introducing an azimuthal separation of $63^{\circ}$ in external space within the same hemifield between the tactile target and the visual distractor). This suggests that the magnitude of the cross-modal congruency effect can be modulated by the distance between the vibrotactile target and the visual distractor within one hemifield, even when this change does not alter the hemisphere to which the stimuli project.

As can be seen in Table 2, cross-modal congruency effects measured for tactile targets at the mobile hand when held in the hands-far posture were not statistically different when visual distractors were presented in the same hemifield (and projecting to the same hemisphere) or in the opposite hemifield (projecting to the opposite hemisphere; 24 vs. $38 \mathrm{msec}$, respectively; $p=.32$ on a paired $t$ test). However, it is possible that our experiment may simply have lacked the power to tease out such a small effect, were one really to be there. Subsequent calculations revealed that the power in our analysis, assuming an expected effect size of $12 \mathrm{msec}$, was 0.17 [ $\delta=.99$, with $N=$ $12, \sigma=50.2, \alpha=.05$; Howell, 1999]. Nevertheless, if any effect does exist, it should be very small in magnitude, and so our main conclusions from this study still stand.

Finally, Experiment 2 also enabled us to examine whether a visual distractor's influence on vibrotactile elevation discrimination responses can depend on the relative proxim- 
ity of the visual distractor to an unstimulated, but visible, hand (cf. di Pellegrino \& Frassinetti, 2000; di Pellegrino, Làdavas, \& Farné, 1997). In other words, is the crossmodal congruency effect elicited by visual distractors on a particular foam block increased if that block happens to be held in one of the participant's hands (even if that hand does not itself receive any vibrotactile stimulation on a given trial)? However, no such evidence emerged from Experiment 2. In particular, when the vibrotactile targets were presented to the fixed hand, there was no significant difference in the magnitude of the cross-modal congruency effect elicited by visual distractors presented in the other hemifield as a function of whether the unstimulated hand was placed adjacent to those visual distractors (mean RT difference $=38 \mathrm{msec}$, mean error difference $=5 \%$ ) or far from them (mean RT difference $=30 \mathrm{msec}$, mean error difference $=5 \%$; see Table 2 ). We may have lacked the power to establish a small (i.e., 8-msec) effect here [power less than $0.17 ; \delta=.53$, with $N=12, \sigma=52.9$, $\alpha=.05$; Howell, 1999], but the more important point is the larger effect of distractor proximity for the stimulated hand. It would appear, then, that simply placing a hand by a foam block, in which a pair of distractor lights is embedded, does not affect the magnitude of the cross-modal congruency effects elicited by those lights, at least when the lights are already positioned within peripersonal space.
Instead, the hand's proximity to the lights matters significantly more for the hand receiving the tactile target.

\section{EXPERIMENT 3}

Having shown that spatial proximity within a hemifield of the vibrotactile target to the visual distractor does play some role in modulating the magnitude of cross-modal congruency effects, we went on, in the next experiment, to examine whether there was also any role at all for the initial hemispheric projection of the target and distractor stimuli in determining cross-modal congruency effects. In Experiment 3, we assessed whether cross-modal congruency effects are based on stimuli that are coded within a relatively high level representation of external space that is updated even when both of the participant's hands are crossed over the midline (see Figure 5), so that the left hand holds the foam block situated on the participant's right, while the right hand holds the foam block situated on the participant's left (cf. Spence, Kingstone, et al., 2001, and Spence, Shore, et al., 2001 for evidence of a failure to remap visuotactile space across the midline for such a crossed posture in a split-brain patient).

In half of the experimental blocks, the participants were again tested in the uncrossed posture adopted in Experiment 1 (same as the hands-close posture of Experiment 2),

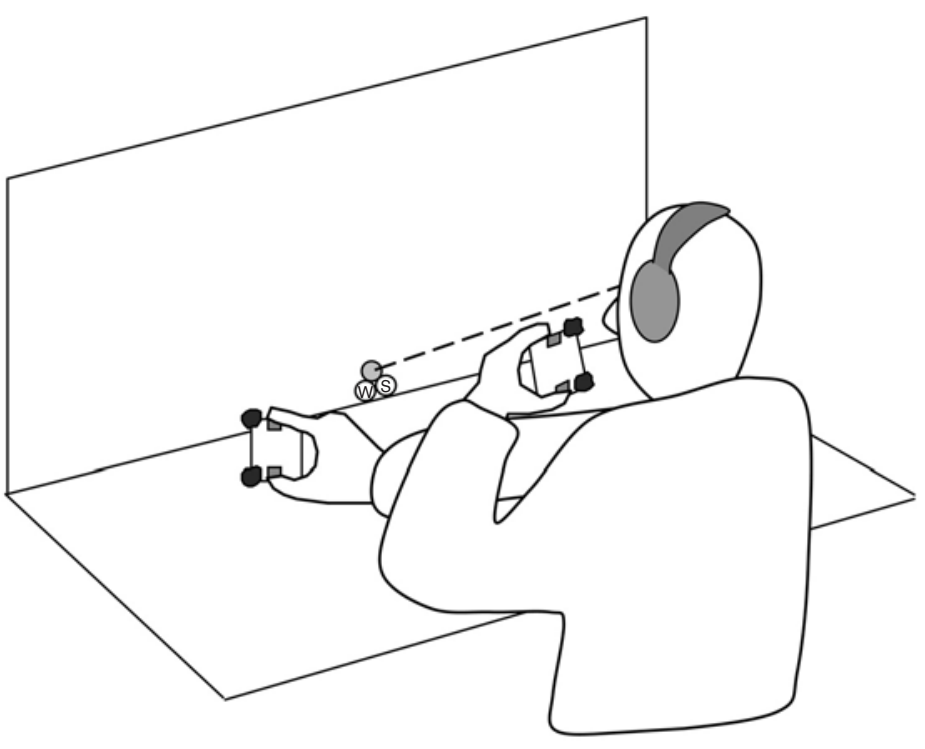

- Distractor light

○ Fixation light
Vibrotactile stimulator

(S) (1) Feedback lights

Figure 5. Schematic view of the apparatus and participant adopting the crossed-hands posture in Experiment 3. As before, the participant held a foam cube in each hand. Two vibrotactile stimulators (shaded rectangles) and two visual distractor lights (filled circles) were embedded within each foam block, by the thumb and the index finger. White noise was presented over headphones, and the eye position of all the participants was monitored (fixation is shown by the dotted line). 
whereas in the remainder of the blocks, the participants were now tested with both of their hands adopting a crossedhands posture. If cross-modal congruency effects are sensitive to changes in posture and the relative locations of visual and tactile stimuli in external space, we predicted that it should be visual distractors close by the current target hand position that would elicit the largest cross-modal congruency effects no matter what the posture adopted (see also Spence, Kingstone, et al., 2001; Spence, Shore, et al., 2001). However, if the initial hemispheric projections of the stimuli do play a significant role in modulating cross-modal congruency effects, we predicted that there should be some difference in the magnitude of the cross-modal congruency effects reported as a function of which hand held a particular pair of vibrotactile stimulators, with reduced effects for crossed hands (since hemisphere and spatial effects should now be in opposition).

Experiment 3 is by no means the first experiment in which the representation of visuotactile space when the hands are placed in a crossed posture has been investigated. Several previous studies have already addressed this general question for the specific case of visuotactile links in both exogenous and endogenous spatial attention (Kennett, Eimer, Spence, \& Driver, 2001; Kennett et al., 2002; Spence, Pavani, \& Driver, 2000). All of these earlier studies showed that the representation of visuotactile peripersonal space is updated when the hands are crossed over the midline. However, it should be noted that the cross-modal cuing effects shown in all those studies were always demonstrated under conditions in which only a single stimulus was presented at any one time (i.e., there were no distractors in these previous studies, whereas the present studies specifically focus on the ability to ignore distractors in a task-irrelevant modality). Importantly, several recent studies have shown that people may find it more difficult to remap vibrotactile and visual stimuli when two stimuli are presented at about the same time than when they are presented sequentially (e.g., Shore, Spry, \& Spence, 2002; Spence, Baddeley, Zampini, James, \& Shore, 2003; Yamamoto \& Kitazawa, 2001). Given that the presentations of the target and the distractor stimuli overlapped in the experiments reported here, and given the many differences between attentional cuing studies and distractor effects involved in the cross-modal congruency task (as also is highlighted in Experiment 1 here), we judged it important to investigate any impact of the crossing of both hands on cross-modal congruency effects.

\section{Method}

Participants. Eight participants ( 6 women and 2 men) took part in this experiment. Their mean age was 24 years, with a range from 20 to 30 years. All were right-handed by self-report and reported normal touch and normal or corrected vision. Each took part in one experimental session, which lasted for approximately $60 \mathrm{~min}$. Three of the participants had taken part in the preceding experiment.

Apparatus, Materials, Design, and Procedure. The apparatus, materials, design, and procedure were the same as those in Experiment 1, with the following exceptions. First, target side was now entirely predictable in every block of trials. We introduced the latter change in design (as compared with the previous experiment, in which target side had always been unpredictable) because we thought a priori that a complete remapping of visuotactile space with hands crossed might be more likely if the participants could focus their spatial attention on a particular side/hand throughout each block of trials. Second, hand posture (both hands crossed and hands uncrossed) was manipulated here.

The three within-subjects factors were the congruency between the elevation of the vibrotactile target and the visual distractor (congruent vs. incongruent), the relative hemisphere to which the target and the distractor stimuli initially projected (same vs. different), and the posture adopted (crossed vs. uncrossed hands). There were two blocks of 15 practice trials, followed by eight test blocks of 64 trials each. Hand posture remained constant for the first four experimental blocks but was changed for the remaining four blocks. Half of the participants started with their hands in the uncrossed posture, whereas the remainder began with their hands in the crossed posture. In each block of trials, equal numbers of vibrotactile targets were presented from each of the two possible target positions (up vs. down) on one particular side/hand. The visual distractors were always equally likely to occur from any one of the four possible distractor positions (two on each side) on each trial.

The procedure was the same as that in Experiment 1, except as follows. On half of the experimental blocks, the participants were required to cross both their hands over the midline. In the crossedhands blocks, they grasped the right foam block between the forefinger and the thumb of their left hand and the left foam block between the forefinger and the thumb of their right hand, at the same eccentricity as before (see Figure 5). The participants were informed at the beginning of each block of trials about the side of the vibrotactile targets with the verbal instruction "attend left" or "attend right." Once again, eye movements were monitored for all the participants to ensure the maintenance of central fixation.

\section{Results}

Trials with incorrect response were discarded from the RT analysis. Due to excessive eye movements (or blink ar-

Table 3

Mean Reaction Times (RTs, in Milliseconds), Their Standard Deviations, Percentages of Errors (\%E), and Mean Cross-Modal Congruency Effects (CCEs) for Vibrotactile Targets in Experiment 3, as a Function of Visual Distractor Hemifield and Posture

\begin{tabular}{|c|c|c|c|c|c|c|c|c|c|}
\hline \multirow{3}{*}{$\begin{array}{c}\text { Relative } \\
\text { Distractor } \\
\text { Hemisphere }\end{array}$} & \multirow[b]{3}{*}{ Posture } & \multicolumn{3}{|c|}{ Congruent Distractor } & \multicolumn{3}{|c|}{ Incongruent Distractor } & & \\
\hline & & \multicolumn{2}{|c|}{ RT } & \multirow[b]{2}{*}{$\% \mathrm{E}$} & \multicolumn{2}{|c|}{ RT } & \multirow[b]{2}{*}{$\% \mathrm{E}$} & \multicolumn{2}{|c|}{ Mean CCE } \\
\hline & & $M$ & $S D$ & & $M$ & $S D$ & & RT & $\% \mathrm{E}$ \\
\hline \multirow[t]{2}{*}{ Same hemisphere } & Uncrossed hands & 440 & 57 & 4 & 502 & 65 & 13 & $62 *$ & $9 * *$ \\
\hline & Crossed hands & 468 & 86 & 4 & 470 & 76 & 6 & 3 & $2 *$ \\
\hline \multirow[t]{2}{*}{ Different hemispheres } & Uncrossed hands & 450 & 60 & 5 & 470 & 56 & 5 & $20 *$ & 0 \\
\hline & Crossed hands & 437 & 70 & 3 & 504 & 62 & 16 & $67 * *$ & $13^{*}$ \\
\hline
\end{tabular}

$* p<.05 . \quad * * p<.01$ 
tifacts), $8.6 \%$ of the trials were removed, and $0.8 \%$ of the trials were removed across all participants because no response was made within 1,500 msec of target onset. Table 3 shows the interparticipant mean cross-modal congruency effects in the RT and error data as a function of the relative hemisphere, posture, and target-distractor congruency. As in Experiment 2, a preliminary within-subjects ANOVA with the factor of target-distractor congruency (2) was conducted on the RT and error data, to evaluate the presence of an overall cross-modal congruency effect. This analysis revealed that the participants responded significantly more rapidly overall on congruent distractor trials $(M=449 \mathrm{msec})$ than on incongruent distractor trials $[M=487 \mathrm{msec} ; F(1,7)=16.7, p<.005]$, as usual. The participants also made fewer errors on congruent trials $(M=4.0 \%)$ than on incongruent distractor trials overall $[M=10.0 \% ; F(1,7)=14.2, p<.007]$, replicating the finding of a basic cross-modal congruency effect, as was found in the two previous experiments.

A within-subjects ANOVA with two factors (relative hemisphere [2] $\times$ posture [2]) was then conducted on the mean cross-modal congruency effects in the RT data. Note that in the present analysis, the levels of the relative hemisphere factor refer to the anatomical hemisphere to which the target and distractor stimuli initially projected. That is, a vibrotactile target presented to the right hand and a visual distractor presented to the right visual field or a vibrotactile target on the left hand and a visual distractor presented to the left visual field constituted the samehemisphere conditions in both the uncrossed- and the crossed-hands conditions.

Analysis of the cross-modal congruency effects in the RT data revealed a significant interaction between relative hemisphere and posture $[F(1,7)=10.4, p<.01]$. When the participant's hands were placed in an uncrossed-hands posture, larger cross-modal congruency effects were observed when the vibrotactile target and the visual distractor stimuli projected to the same hemisphere $(M=63 \mathrm{msec})$ from the same side than when they were projected to different hemispheres $(M=20 \mathrm{msec}, p<.01)$ from opposite hemifields. This pattern of results was completely reversed when the hands were crossed over the midline, with larger cross-modal congruency effects now being reported when the target and the distractor stimuli initially projected to different cerebral hemispheres $(M=67 \mathrm{msec})$ than when they projected to the same hemisphere $(M=$ $3 \mathrm{msec}, p<.01)$. None of the other terms in the analysis of the RT data reached significance (all $\left.F_{\mathrm{S}}<1.5\right)$.

A similar analysis of the error data also revealed a significant interaction between relative hemisphere and posture $[F(1,7)=8.8, p<.02]$. When the participant's hands were placed in an uncrossed-hands posture, cross-modal congruency effects were again significantly larger when the target and the distractor stimuli projected to the same hemisphere $(M=9.0 \%)$ from the same side of space than when they were initially projected to different hemispheres $(M=-0.1 \%, p<.02)$. By contrast, when the participant's hands were placed in the crossed posture, larger cross- modal congruency effects were now observed when the target and the distractor stimuli initially projected to different cerebral hemispheres $(M=12.9 \%)$ than when they projected to the same hemisphere $(M=2.4 \%, p<.01)$. The main effect of posture was borderline significant $[F(1,7)=4.47, p=.07]$ but was subsidiary to the twoway interaction. None of the other terms in this analysis of the error data approached significance (all $F_{\mathrm{S}}<1$ ).

\section{Discussion}

Experiment 3 once again replicated the existence of the basic cross-modal congruency effects observed in the two previous experiments. When the participant's hands were placed in an uncrossed posture (analogous to the posture adopted in Experiment 1 and to the hands-close condition of Experiment 2), visual distractors presented from the same foam block (and initially projecting to the same hemisphere) as the vibrotactile targets elicited larger crossmodal congruency effects than did visual distractors presented from the foam block situated on the opposite side to the vibrotactile targets (and projecting initially to the other hemisphere), again replicating the basic "spatial" modulation of the cross-modal congruency effect, as was reported in the previous two experiments.

The new finding to emerge from Experiment 3 comes from the crossed-hands condition, in which the visual and the vibrotactile stimuli that were initially projected to the same cerebral hemisphere (e.g., a touch to the right hand and a flash in the right visual field, both projecting initially to the left hemisphere) were now actually positioned on opposite sides in external space (e.g., the crossed right hand was adjacent to visual distractors in the left visual field), and vice versa for stimuli that were on "different sides" in terms of their initial hemispheric projections but now on the same side in external space. When analyzed in terms of their initial hemispheric projections, the results showed a complete reversal of the cross-modal congruency effect across the two hand postures, with a larger cross-modal congruency effect for different-hemisphere than for same-hemisphere trials when the hands were crossed over the midline. In other words, there was a complete remapping of visuotactile space for cross-modal congruency effects when the participants crossed their hands over the midline.

The results of Experiment 3 accord with the findings reported in a number of previous behavioral and electrophysiological studies of cross-modal links in spatial attention between vision and touch (e.g., Kennett et al., 2001, 2002; Spence, Pavani, \& Driver, 2000). Here, the remapping result is extended for the first time to cross-modal distractor congruency effects from task-irrelevant stimuli in a task-irrelevant modality. They contrast with the partial remapping reported in Spence et al.'s (2003) visualtactile temporal order judgment studies (cf. Röder, Rösler, \& Spence, 2004; Shore et al., 2002; Yamamoto \& Kitazawa, 2001).

We wondered what role, if any, continuous visual information regarding posture may have played in main- 
taining the updated representation of visuotactile space in the hands-crossed posture of Experiment 3. We therefore conducted a final experiment in which we compared the pattern of cross-modal congruency effects observed when participants adopted the crossed-hands posture with the room lights illuminated throughout a block of trials (so that the participants could see their arms continuously, as in all the previous experiments reported here) with those seen when all ambient illumination was extinguished and the blocks of trials were conducted in complete darkness (so that the participants no longer had any direct visual information regarding the position of their limbs). The results showed that the complete remapping of visuotactile peripersonal space when the participant's hands were crossed over the midline was maintained regardless of whether the participants could see their hands or not. ${ }^{2}$ In fact, our participants' ability to see their hands (the hand visibility factor) had absolutely no significant effect on performance whatsoever in this follow-up study. This result suggests that a posture-dependent representation of peripersonal visuotactile space can be maintained in the absence of continuous visual cues regarding current limb position.

It should, however, be noted here that the participants would have been able to see their hands at the very start of each block of trials in this follow-up study. Moreover, the fact that the participants knew that the distractor lights were situated on the two foam cubes held in their hands and the fact that the visual distractors were illuminated shortly (i.e., $30 \mathrm{msec}$ ) before the target vibrations occurred means that some residual visual cues regarding hand position were occasionally available in the follow-up. Nevertheless, running this follow-up in an otherwise dark setting did eliminate the continuously available sight of one's limbs in the crossed posture that had been available in Experiment 3.

\section{GENERAL DISCUSSION}

A number of conclusions can be drawn from the experiments reported here. First, a robust cross-modal congruency effect was demonstrated (in terms of both the RT and the error data) in all the experiments. This result highlights our participants' inability to completely ignore irrelevant inputs presented to one sensory modality (vision here) while attempting to respond selectively just to stimuli presented in another relevant target modality (touch here; see Marks, 2004, and Pashler, 1998, on this general topic). Second, the magnitude of the visual-tactile crossmodal congruency effect in all of our experiments was modulated by the relative spatial location of the tactile target and the visual distractor stimuli in external space. In particular, the participants found it harder to ignore irrelevant visual distractors when they were presented from the same foam block as the vibrotactile targets (i.e., from the same lateral location in space) than when they were presented from different foam blocks (i.e., from different lateral spatial locations, in separate hemifields; see Figure 2A). Such spatial modulation of cross-modal interference (or distractor congruency) effects has received relatively little re- search to date, despite the long-standing work on distractor interference effects within single modalities (e.g., B. A. Eriksen \& C. W. Eriksen, 1974). Jones and Hapeshi (1991) and Spence, Ranson, and Driver (2000) published some of the initial cross-modal research on the difficulty of ignoring distractors in a second modality (although see also Pavani et al., 2000; Maravita, Spence, Kennett, \& Driver, 2002; Maravita, Spence, Sergent, \& Driver, 2002), with both groups reporting some spatial modulation of audiovisual cross-modal distractor interference, as we show here for a tactile-visual situation.

Experiment 1 also demonstrated that the participants found it harder to ignore irrelevant visual distractors (i.e., they made significantly more errors) when their onset slightly preceded that of the vibrotactile targets than when the two stimuli were presented simultaneously or when the onset of the target preceded that of the distractor by $30 \mathrm{msec}$ (see Figure 2B). This result is in line with the results of a number of other previous studies of single sensory modalities that have shown that many different kinds of distractor interference effect are modulated by the relative timing of the processing of the relevant and irrelevant stimulus information (e.g., Dyer, 1971; C. W. Eriksen \& Hoffman, 1972; Hommel, 1993b; see also Lu \& Procter, 1995).

We also found that making the target side/hand predictable in Experiment 1 facilitated the overall latencies of tactile discrimination responses, when performance was compared with that in the unpredictable target-side blocks (see Table 1). Somewhat surprisingly, however, this effect of endogenous (or voluntary) focusing of tactile spatial attention on a particular side/hand had no significant effect on the magnitude of the overall cross-modal congruency effects reported (i.e., it did not affect the ease with which the participants ignored the irrelevant visual distractors on one side or the other; see Figure 2C) or on the spatial modulation of cross-modal congruency effects that was equivalent to that found when target side was predictable. This particular cross-modal result contrasts with the results of a number of previous intramodal studies in which focusing spatial attention on a particular location has been shown to reduce, or even to eliminate, distractor interference effects from task-irrelevant stimuli at other locations (e.g., Besner \& Stolz, 1999; Soto-Faraco, Ronald, \& Spence, in press; see also Yantis, 1996, 2000).

In our second experiment, we examined any effect of posture change on the magnitude of the cross-modal congruency effects elicited by visual distractors presented from the same locations in front of the participants as before (in the same retinal positions as the distractor lights in Experiment 1). One of the participant's hands was always held close to the distractor lights in the same hemifield, while the other hand was either held by the distractor lights on the other side of fixation (the hands-close posture) or positioned more eccentrically on that side (i.e., $63^{\circ}$ from the distractor lights within that same hemifield; the hands-far posture). When the hand receiving the vibrotactile target (i.e., the target hand) was placed close to visual distractors within the same hemifield in Experi- 
ment 2, cross-modal congruency effects were much larger than when the target hand was placed far from the visual distractors within that same hemifield (compare Figures 4A and 4B). Crucially, this modulation of the cross-modal congruency effect as a function of the proximity of the tactile target and the visual distractor within one hemifield in external space occurred despite the fact that the vibrotactile stimuli were applied to the same tactile receptors and, likewise, the possible visual distractors occupied exactly the same retinal positions, no matter what posture was adopted. Our results add to a growing body of evidence highlighting the multisensory nature of spatial judgments concerning vibrotactile stimuli. They also provide further support for the growing body of empirical evidence showing that the perception of tactile stimuli tends to incorporate information about the current spatial position of tactile receptors in external space (cf. Driver \& Grossenbacher, 1996; Kennett et al., 2001; Kennett et al., 2002; Shore et al., 2002, 2004; Soto-Faraco, Ronald, \& Spence, in press; Yamamoto \& Kitazawa, 2001), even though posture may be completely irrelevant to the participant's task.

The results of Experiment 2 also revealed that the crossmodal congruency effects elicited by a particular pair of visual distractor stimuli were not significantly affected by whether the nontarget (currently tactually unstimulated) hand was placed close to or far from the visual distractors (i.e., either holding the foam block directly next to the distractor lights or positioned at a far more eccentric location, $63^{\circ}$ further away on the same side; compare Figures 4C and 4D). This finding may be particularly relevant for the interpretation of some previous evidence showing that cross-modal congruency effects elicited by visual distractors far from the participant's hands can increase when a pair of rubber hands (e.g., Austen et al., 2001; Pavani et al., 2000; see also Walton \& Spence, 2004) or the mirrorreflection of the participant's hands (Maravita, Spence, Sergent, \& Driver, 2002) appear to grasp the distractor lights. Since distractor lights are apparently not made more salient merely by their proximity to a currently unstimulated hand (as is shown for the first time here), the increased cross-modal congruency effects in those other studies seem likely to reflect genuine effects on the crossmodal representation of peripersonal space, due to visuoproprioceptive illusions (e.g., Pavani et al., 2000) or to repeated identification of mirror-reflected body parts as the participant's own (Holmes, Crozier, \& Spence, 2004; Maravita, Spence, Sergent, \& Driver, 2002).

In Experiment 3, we investigated any effects of a more extreme change of posture - namely, crossing the handson the spatial modulation of cross-modal congruency effects. Our results suggested a remapping of visuotactile space when the hands were crossed, since it was the relative spatial location of target and distractor stimuli in external space, and not simply the cerebral hemisphere to which the stimuli projected initially (cf. Kinsbourne \& Cook, 1971; Kinsbourne \& Hicks, 1978; Passarotti et al., 2002), that determined the magnitude of cross-modal distractor congruency effects. A follow-up experiment fur- ther showed that this remapping of visuotactile space took place even when no continuous visual cues regarding limb position were provided. Such results suggest that the remapping of visuotactile space following posture change might involve proprioceptive cues regarding limb position. Our results are thus consistent with a number of previous findings according to which cross-modal links in visuotactile attention are updated to take current posture into account when the hands are crossed over the midline (e.g., Kennett et al., 2001, Kennett et al., 2002; Spence, Pavani, \& Driver, 2000). These results are also consistent with the findings emerging from a number of neuropsychological studies of patients exhibiting cross-modal extinction (e.g., di Pellegrino et al., 1997; see Làdavas, 2002, and Làdavas \& Farnè, 2004, for reviews). Here, we show for the very first time that such updating of visual-tactile space occurs even for distractor effects arising crossmodally from an entirely task-irrelevant modality (vision here).

However, it is also worth noting that, in contrast to the findings reported here, a number of researchers have actually documented some impairment in certain behavioral responses to tactile stimuli when participants adopt a crossed-hands, as opposed to an uncrossed-hands, posture (see, e.g., Driver \& Spence, 1998; Groh \& Sparks, 1996; Pierson, Bradshaw, Meyer, Howard, \& Bradshaw, 1991; Röder et al., 2004; Shore et al., 2002; Yamamoto \& Kitazawa, 2001). Interestingly, such behavioral impairments have typically been demonstrated under conditions in which the participants were required either to make a simple speeded detection response or, more commonly, to make some form of left-right discrimination response. It is possible that a confusion between the coding of leftright responses in an allocentric versus an egocentric frame of reference may have accounted for the behavioral impairments seen in at least some of the studies on left-right judgments (e.g., Hommel, 1993a; Riggio, Gawryszewski, \& Umiltà, 1986; Shore et al., 2002; Yamamoto \& Kitazawa, 2001). By contrast, the left-right manipulations of hand position in the present study were always orthogonal to the up-down direction in which the participants had to make their elevation discrimination responses, hence potentially avoiding any such confusion.

Taken together, the results of the experiments reported here show that spatial modulation of the cross-modal congruency effects found in normals arises in representation(s) of space that show some appropriate remapping of one sensory modality with respect to the other (in terms of the combination of receptor stimulations that produce maximal effects) across changes in posture (e.g., such that the right hand becomes the effective partner of the left visual field, and vice-versa, when the hands are crossed, whereas the reverse pairings apply for uncrossed hands in Experiment 3; see Table 3). This conclusion makes considerable functional sense. In daily life, our posture changes frequently (e.g., during eye and hand movements), so that which particular tactile receptors represent the same external location in space as that represented by particular 
visual receptors will continuously vary. If the modulation of the cross-modal congruency effects were to reflect a fixed anatomical mapping between the senses, this could not always serve the adaptive function of relating sensory information from a common external source across the modalities. Achieving this requires that the cross-modal links in information processing should operate within more abstract spatial coordinates (as we have found) that treat a common external location as the same across the modalities regardless of which particular receptors within each sensory modality currently correspond to that common external position. This should facilitate cross-modal integration for stimuli from the same or neighboring locations in external space (Spence \& Driver, 2004; Stein \& Meredith, 1993), but as was found here, this may be at the cost of making distractor information from such locations harder to ignore. The robustness of the visual-tactile crossmodal congruency task as an index of the apparent colocation of visual and tactile stimuli in space having been demonstrated, one particularly interesting question for future research may be to determine how quickly this remapping of visuotactile space can occur following continuous postural changes: Does it, for example, occur immediately, or does remapping across postures take time to arise? Although there would obviously be evolutionary advantages to the immediate updating of the visuotactile representation of space following posture change, recent studies have shown that, at least for the case of the "plastic" modification of peripersonal space seen following tool use, such changes may take some time to occur (Maravita, Spence, Kennett, \& Driver, 2002; see also Holmes, Calvert, $\&$ Spence, 2004).

In addition to making functional sense, our results may also accord with recent neuroscience findings of single units that appear to represent common external locations in vision and in touch (e.g., Duhamel, Colby, \& Goldberg, 1991; Rizzolatti, Scandolara, Matelli, \& Gentilucci, 1981) and to show appropriate remapping between these senses across changes in posture. For instance, Graziano, Gross, and their colleagues have observed neurons in parietal area $7 \mathrm{~b}$, the ventral premotor cortex, and the putamen that respond to both tactile and visual stimulation (see Graziano \& Botvinick, 2002, for a recent review). These multimodal neurons typically have receptive fields for corresponding sectors of space in the two sensory modalities. For instance, a unit with a tactile receptive field on the right hand would typically have a visual receptive field close to that hand. Critically, such visual receptive fields have been shown to follow the corresponding body part around to some extent as it is moved (whether passively or actively), even when the eyes do not move. These units therefore exhibit remapping in terms of which visual and tactile receptors serve to stimulate the neuron. Although it seems tempting to suppose that similar units might be involved in generating the spatial modulation of the crossmodal congruency effects that we observed behaviorally in the present study (cf. Làdavas, 2002; Spence, Kingstone, et al., 2001), it is important to note that the exact func- tional roles of such neurons has yet to be determined empirically (see Maravita et al., 2003). Functional imaging in humans of paradigms related to the present work could shed light on this issue.

\section{Spatial Constraints on Cross-Modal Focused and Divided Attention}

The results of the experiments reported here show that increasing the separation between relevant and irrelevant stimuli in external space can allow for the more efficient selective processing of information, even when the stimuli in question are presented in different sensory modalities. This extends the spatial separation principle, traditionally found for efficient selective attention, from the strictly unimodal settings in which it has previously been studied (see, e.g., Chan, Merrifield, \& Spence, 2004; B. A. Eriksen \& C. W. Eriksen, 1974) to a multisensory case. It is a well-known generalization (e.g., Kahneman \& Treisman, 1984) that factors that make the division of attention more difficult (such as the presentation of two information sources from different positions) often tend to make selective attention easier, as was found here when irrelevant visual stimuli were placed in very different locations from the relevant vibrotactile target stimuli. When the present focused, or selective, attention results are taken together with some of our previous findings regarding the spatial constraints on the division of visual and tactile attention across different spatial locations (Spence, Pavani, \& Driver, 2000), it would appear that there are constraints on the effective deployment of spatial attention that operate cross-modally that are similar to those previously shown to operate intramodally. Moreover, similar spatial constraints on focused and divided attention have now also been shown to exist cross-modally between both audition and vision (e.g., Driver \& Spence, 1994; Spence \& Driver, 1996; Spence, Ransom, \& Driver, 2000; although see Soto-Faraco, Morein-Zamir, \& Kingstone, in press) and between audition and touch (see, e.g., Lloyd, Shore, Spence, \& Calvert, 2003; Merat, Spence, Lloyd, Withington, \& McGlone, 1999), suggesting that efficient division of attention across stimuli at neighboring external locations, but efficient selective attention when targets and distractors are at very different locations, may be a rather general rule reflecting a fundamental constraint on human information processing (see Driver \& Spence, 2004, for a recent review).

\section{Do Cross-Modal Congruency Effects Reflect Perception, Response Competition, or Both?}

The slowing of performance on incongruent distractor trials might conceivably reflect a strictly perceptual interaction between vision and touch (cf. Austen et al., 2001; Marks, 2004), such as a mislocation of one stimulus toward the other (i.e., a ventriloquism-like effect; see Bertelson \& de Gelder, 2004). In principle, this could make it more difficult to determine the true elevation of the target event when the distractor is presented from an incongruent, rather than from a congruent, elevation. However, we 
think that such multisensory perceptual interactions inducing strict perceptual mislocalization may account for only a small component of the overall cross-modal congruency effects reported in the present study (see the Appendix for some empirical evidence on this point).

Alternatively, it could be argued that there may be some spatial-cuing explanation for the existence of the crossmodal congruency effect. Indeed, the fact that the largest cross-modal congruency effects were reported when the distractor stimuli were presented shortly before the target stimuli (see Experiment 1) means that the paradigm used in the majority of the experiments reported here bears some methodological similarity to the design of many cross-modal precuing studies of spatial attention, where a cuing event is presented shortly before a target event in a different sensory modality (e.g., Chong \& Mattingley, 2000; Kennett et al., 2001; Kennett et al., 2002; Posner, 1980; Spence \& Driver, 1997). However, we have argued previously (see Spence, Shore, et al., 2001) that although cross-modal attentional spatial-cuing effects may have some overall effect on performance in the cross-modal congruency task (such as, perhaps, facilitating all responses to targets on same-side trials, relative to differentside trials), they cannot, on their own, account for the congruency effect itself. In particular, they fail to account for the fact that the spatial modulation of the congruency effect typically results from a slowing of response latencies on incongruent same-side distractor trials, with performance on congruent trials showing little difference as a function of the relative side from which the distractor is presented (i.e., for the worse performance on incongruent trials, when compared with congruent trials). A traditional precuing effect from the distractor should speed sameside conditions, but instead slower performance is found, especially for incongruent same-side trials.

Our own view is that the cross-modal congruency effect is likely to primarily reflect response competition between the response tendencies elicited by the target and the distractor on incongruent trials (see Coles, Gratton, Bashore, C. W. Eriksen, \& Donchin, 1985; B. A. Eriksen \& C. W. Eriksen, 1974). It seems likely that the presentation of both the target and the distractor stimuli will prime the up or down response(s) associated with the elevation at which they are presented. Given that the distractor will prime the incorrect response on incongruent trials, this might lead to a slowing of responses to the target, attributable to the time taken to overcome the incongruent (i.e., "inappropriate") response tendency (see also Dyer, 1971; Hommel, 1993b). In fact, the slowest responses in cross-modal congruency experiments are typically reported on trials in which the visual distractor is presented from the same side as the vibrotactile target, but at an incongruent elevation. By contrast, a participant's performance of congruent trials might be expected to show some degree of response facilitation, since the target and the distractor stimuli would both prime the same, "correct" response (see also Marks, 2004, for a detailed review of the various factorssemantic, perceptual, and synaesthetic - thought to un- derlie various kinds of congruency effects). But note that the critical point of the present experiments still remains regardless of all these considerations - namely, that the cross-modal congruency effects depend strongly on the relative location of tactile target and visual distractor stimuli in external space. They do not depend solely on whether the target and the distractor agree or disagree in terms of the up-down response associated with them, since this response competition is much larger when the target and the distractor come from similar external locations. This shows that an incongruent visual distractor intrudes more into tactile judgments when arising from a nearby location.

Whatever the underlying cause(s) of the cross-modal congruency effect turn out to be, the spatial modulation of the effect has been shown here to provide a sensitive and robust measure of what can constitute the same location across vision and touch. Having gained a better understanding of the basic constraints on this effect, we are, therefore, now in a better position to move forward in using the cross-modal congruency task to address many important questions related to the multisensory construction of peripersonal space (see Maravita et al., 2003, for a review of such issues).

\section{REFERENCES}

Austen, E. L., Soto-Faraco, S., Pinel, J. P. J., \& Kingstone, A. F. (2001). Virtual body effect: Factors influencing visual-tactile integration. Abstracts of the Psychonomic Society, 6, 2.

BANICH, M. T. (1998). The missing link: The role of interhemispheric interaction in attentional processing. Brain \& Cognition, 36, 128-157.

Bertelson, P., \& De Gelder, B. (2004). The psychology of multimodal perception. In C. Spence \& J. Driver (Eds.), Crossmodal space and crossmodal attention (pp. 141-177). Oxford: Oxford University Press.

Besner, D., \& Stolz, J. A. (1999). What kind of attention modulates the Stroop effect? Psychonomic Bulletin \& Review, 6, 99-104.

Bolognini, N., Frassinetti, F., \& LÀdavas, E. (2003). Acoustical vision of below threshold stimuli: Interaction among spatially converging audio-visual inputs. Manuscript submitted for publication.

Bradshaw, J. L., Howard, M. J., Pierson, J. M., Phillips, J., \& BradSHAW, J. A. (1992). Effects of expectancy and attention in vibrotactile choice reaction time tasks. Quarterly Journal of Experimental Psychology, 44A, 509-528.

Chan, J. S., Merrifield, K., \& Spence, C. (2004). Auditory spatial attention assessed in a flanker interference task. Manuscript submitted for publication.

Chong, T., \& Mattingley, J. B. (2000). Preserved cross-modal attentional links in the absence of conscious vision: Evidence from patients with primary visual cortex lesions [Abstract]. Journal of Cognitive Neuroscience, 12(Suppl.), 38.

Coles, M. G. H., Gratton, G., Bashore, T. R., Eriksen, C. W., \& DONCHIN, E. (1985). A psychophysiological investigation of the continuous flow model of human information processing. Journal of Experimental Psychology: Human Perception \& Performance, 11, 529-553.

di Pellegrino, G., \& Frassinetti, F. (2000). Direct evidence from parietal extinction of enhancement of visual attention near a visible hand. Current Biology, 10, 1475-1477.

di Pellegrino, G., Làdavas, E., \& Farné, A. (1997). Seeing where your hands are. Nature, $\mathbf{3 8 8}, 730$.

Driver, J., \& GrosSENBACHER, P. G. (1996). Multimodal spatial constraints on tactile selective attention. In T. Inui \& J. L. McClelland (Eds.), Attention and performance XVI: Information integration in perception and communication (pp. 209-235). Cambridge, MA: MIT Press.

Driver, J., \& Spence, C. J. (1994). Spatial synergies between auditory 
and visual attention. In C. Umiltà \& M. Moscovitch (Eds.), Attention and performance $X V$ : Conscious and nonconscious information processing (pp. 311-331). Cambridge, MA: MIT Press.

Driver, J., \& Spence, C. (1998). Crossmodal links in spatial attention. Philosophical Transactions of the Royal Society of London: Series B, 353, 1319-1331.

DRIVER, J., \& SPENCE, C. (2004). Crossmodal spatial attention: Evidence from human performance. In C. Spence \& J. Driver (Eds.), Crossmodal space and crossmodal attention (pp. 179-220). Oxford: Oxford University Press.

Duhamel, J.-R., Colby, C. L., \& Goldberg, M. E. (1991). Congruent representations of visual and somatosensory space in single neurons of monkey intra-parietal cortex (area VIP). In J. Paillard (Ed.), Brain and space (pp. 223-236). New York: Oxford University Press.

DYER, F. N. (1971). The duration of word meaning responses: Stroop interference for different preexposures of the word. Psychonomic Science, 25, 229-231.

EIMER, M. (2004). Electrophysiology of human crossmodal spatial attention. In C. Spence \& J. Driver (Eds.), Crossmodal space and crossmodal attention (pp. 221-245). Oxford: Oxford University Press.

EIMER, M., \& Driver, J. (2000). An event-related brain potential study of cross-modal links in spatial attention between vision and touch. Psychophysiology, 37, 697-705.

ERIKSEN, B. A., \& ERIKSEN, C. W. (1974). Effects of noise letters upon the identification of a target letter in a nonsearch task. Perception \& Psychophysics, 16, 143-149.

Eriksen, C. W., \& Hoffman, J. E. (1972). Temporal and spatial characteristics of selective encoding from visual displays. Perception \& Psychophysics, 12, 201-204.

Frassinetti, F., Bolognini, N., \& Làdavas, E. (2002). Enhancement of visual perception by crossmodal visuo-auditory interaction. $\underline{E x}$ perimental Brain Research, 147, 332-343.

FrIEDMAN, A., \& Polson, M. C. (1981). Hemispheres as independent resource systems: Limited-capacity processing and cerebral specialization. Journal of Experimental Psychology: Human Perception \& Performance, 7, 1031-1058.

GraZIANO, M. S. A., \& Botvinick, M. M. (2002). How the brain represents the body: Insights from neurophysiology and psychology. In W. Prinz \& B. Hommel (Eds.), Common mechanisms in perception and action: Attention and performance XIX (pp. 136-157). Oxford: Oxford University Press.

GroH, J. M., \& Sparks, D. L. (1996). Saccades to somatosensory targets: 1. Behavioral characteristics. Journal of Neurophysiology, 75, 412-427.

Holmes, N., Calvert, C., \& Spence, C. (2004). Does tool-use extend visuotactile peripersonal space? Evidence from the crossmodal congruency task. Manuscript submitted for publication.

Holmes, N., Crozier, G., \& SPEnCE, C. (2004). When mirrors lie: "Visual capture" of arm position impairs reaching performance. Cognitive, Affective, \& Behavioral Neuroscience, 4, 194-201.

Holmes, N., \& SPENCE, C. (2004). The body schema and the multisensory representation(s) of peripersonal space. Cognitive Processing, 5, 94-105.

Hommel, B. (1993a). Inverting the Simon effect by intention: Determinants of direction and extent of effects of irrelevant spatial information. Psychological Research, 55, 270-279.

HommeL, B. (1993b). The relationship between stimulus processing and response selection in the Simon task: Evidence for a temporal overlap. Psychological Research, 55, 280-290.

HoweLL, D. C. (1999). Fundamental statistics for the behavioural sciences. Pacific Grove, CA: Duxbury.

Jones, D. M., \& HAPESHI, K. (1991). Final report of the contract: Information-processing under high workload. Army Personnel Research Establishment, Farnborough, U.K.

Kahneman, D., \& Treisman, A. (1984). Changing views of attention and automaticity. In R. Parasuraman \& D. R. Davies (Eds.), Varieties of attention (pp. 26-61). San Diego: Academic Press.

Kennett, S., Eimer, M., Spence, C., \& Driver, J. (2001). Tactilevisual links in exogenous spatial attention under different postures: Convergent evidence from psychophysics and ERPs. Journal of Cognitive Neuroscience, 13, 462-478.

KenNetT, S., SPEnCE, C., \& Driver, J. (2002). Visuo-tactile links in covert exogenous spatial attention remap across changes in unseen hand posture. Perception \& Psychophysics, 64, 1083-1094.

Kinsbourne, M., \& CoOK, J. (1971). Generalized and lateralized effects of concurrent verbalization on a unimanual skill. Quarterly Journal of Experimental Psychology, 23, 341-345.

Kinsbourne, M., \& Hicks, R. E. (1978). Functional cerebral space: A model for overflow, transfer and interference effects in human performance: A tutorial review. In J. Requin (Ed.), Attention and performance VII (pp. 345-362). Hillsdale, NJ: Erlbaum.

LÀDAVAS, E. (2002). Functional and dynamic properties of visual peripersonal space. Trends in Cognitive Sciences, 6, 17-22.

LÀdAVAS, E., \& FARNÈ, A. (2004). Neuropsychological evidence for multimodal representations of space near specific body parts. In C. Spence \& J. Driver (Eds.), Crossmodal space and crossmodal attention (pp. 69-98). Oxford: Oxford University Press.

Lloyd, D. M., Merat, N., McGlone, F., \& Spence, C. (2003). Crossmodal links between audition and touch in covert endogenous spatial attention. Perception \& Psychophysics, 65, 901-924.

Lloyd, D. M., Shore, D. I., Spence, C., \& Calvert, G. A. (2003). Multisensory representation of limb position in human premotor cortex. Nature Neuroscience, 6, 17-18.

Lu, C.-H., \& Procter, R. W. (1995). The influence of irrelevant location information on performance: A review of the Simon and spatial Stroop effects. Psychonomic Bulletin \& Review, 2, 174-207.

Maravita, A., SPEnCE, C., \& Driver, J. (2003). Multisensory integration and the body schema: Close to hand and within reach. Current Biology, 13, R531-R539.

Maravita, A., Spence, C., Kennett, S., \& Driver, J. (2002). Tool-use changes multimodal spatial interactions between vision and touch in normal humans. Cognition, 83, B25-B34.

Maravita, A., Spence, C., Sergent, C., \& Driver, J. (2002). Seeing your own touched hands in a mirror modulates cross-modal interactions. Psychological Science, 13, 350-356.

MARKs, L. E. (2004). Cross-modal interactions in speeded classification. In G. Calvert, C. Spence, \& B. E. Stein (Eds.), Handbook of multisensory processes (pp. 85-105). Cambridge, MA: MIT Press.

Merat, N., Spence, C., Lloyd, D. M., Withington, D. J., \& MCGlone, F. (1999). Audiotactile links in focused and divided spatial attention. Society for Neuroscience Abstracts, 25, 1417.

Merola, J. L., \& Liederman, J. (1985). Developmental changes in hemispheric independence. Child Development, 56, 1184-1194.

Pashler, H. E. (1998). The psychology of attention. Cambridge, MA: MIT Press.

Passarotti, A. M., Banich, M. T., Sood, R. K., \& Wang, J. M. (2002). A generalized role of interhemispheric interaction under attentionally demanding conditions: Evidence from the auditory and tactile modality. Neuropsychologia, 40, 1082-1096.

Pavani, F., \& CASTIELlo, U. (2004). Binding personal and extrapersonal space through body shadows. Nature Neuroscience, 7, 13-14.

Pavani, F., Spence, C., \& Driver, J. (2000). Visual capture of touch: Out-of-the-body experiences with rubber gloves. Psychological Science, 11, 353-359.

PICK, H. L., JR., WARREN, D. H., \& HAY, J. C. (1969). Sensory conflict in judgments of spatial direction. Perception \& Psychophysics, 6, 203-205.

Pierson, J. M., Bradshaw, J. L., Meyer, T. F., Howard, M. J., \& Bradshaw, J. A. (1991). Direction of gaze during vibrotactile choice reaction time tasks. Neuropsychologia, 29, 925-928.

PosNer, M. I. (1980). Orienting of attention. Quarterly Journal of Experimental Psychology, 32, 3-25.

Riggio, L., GaWryszewsKI, L. DE G., \& Umiltà, C. (1986). What is crossed in crossed-hand effect? Acta Psychologica, 62, 89-100.

Rizzolatti, G., Fadiga, L., Fogassi, L., \& Gallese, V. (1997). The space around us. Science, 277, 190-191.

Rizzolatti, G. C., Scandolara, M., Matelli, M., \& Gentilucci, M. (1981). Afferent properties of periarcuate neurons in macaque monkeys: II. Visual responses. Behavioural Brain Research, 2, 147-163.

RÖDER, B., RöSLER, F., \& SPENCE, C. (2004). Early vision impairs tactile perception in the blind. Current Biology, 14, 121-124.

Shore, D. I., Spry, E., \& Spence, C. (2002). Confusing the mind by crossing the hands. Cognitive Brain Research, 14, 153-163. 
Shore, D. I., Spry, E., \& Spence, C. (2004). Spatial modulation of tactile temporal order judgments. Manuscript submitted for publication.

Soto-Faraco, S., Morein-Zamir, S., \& Kingstone, A. (in press). On audiovisual spatial synergy: The fragility of the phenomenon. Perception \& Psychophysics.

Soto-Faraco, S., Ronald, A., \& Spence, C. (in press). Tactile selective attention and body posture: Assessing the contribution of vision and proprioception. Perception \& Psychophysics.

SPENCE, C. (2001). Crossmodal attentional capture: A controversy resolved? In C. Folk \& B. Gibson (Eds.), Attention, distraction and action: Multiple perspectives on attentional capture (pp. 231-262). Amsterdam: Elsevier Science.

Spence, C. (2002). Multimodal attention and tactile informationprocessing. Behavioural Brain Research, 135, 57-64.

Spence, C., Baddeley, R., Zampini, M., JAMEs, R., \& Shore, D. I. (2003). Multisensory temporal order judgments: When two locations are better than one. Perception \& Psychophysics, 65, 318-328.

Spence, C., \& Driver, J. (1996). Audiovisual links in endogenous covert spatial attention. Journal of Experimental Psychology: Human Perception \& Performance, 22, 1005-1030.

SPENCE, C., \& DRIVER, J. (1997). Audiovisual links in exogenous covert spatial orienting. Perception \& Psychophysics, 59, 1-22.

Spence, C., \& Driver, J. (Eds.) (2004). Crossmodal space and crossmodal attention. Oxford: Oxford University Press.

Spence, C., Kingstone, A., Shore, D. I., \& Gazzaniga, M. S. (2001). Representation of visuotactile space in the split brain. Psychological Science, 12, 90-93.

SpEnce, C., McDonald, J., \& Driver, J. (2004). Exogenous spatialcuing studies of human crossmodal attention and multisensory integration. In C. Spence \& J. Driver (Eds.), Crossmodal space and crossmodal attention (pp. 277-320). Oxford: Oxford University Press.

Spence, C., Pavani, F., \& Driver, J. (1998). What crossing the hands can reveal about crossmodal links in spatial attention. Abstracts of the Psychonomic Society, 3, 13.

SPence, C., Pavani, F., \& Driver, J. (2000). Crossmodal links between vision and touch in covert endogenous spatial attention. Journal of Experimental Psychology: Human Perception \& Performance, 26, 1298-1319.

SPENCE, C., RANSON, J., \& DRIVER, J. (2000). Cross-modal selective attention: On the difficulty of ignoring sounds at the locus of visual attention. Perception \& Psychophysics, 62, 410-424.

Spence, C., Shore, D. I., GazZaniga, M. S., Soto-Faraco, S., \& KINGSTONE, A. (2001). Failure to remap visuotactile space across the midline in the split-brain. Canadian Journal of Experimental Psychology, 55, 135-142.

Spence, C., Shore, D. I., \& Klein, R. M. (2001). Multisensory prior entry. Journal of Experimental Psychology: General, 130, 799-832.

Stein, B. E., \& Meredith, M. A. (1993). The merging of the senses. Cambridge, MA: MIT Press.

Townsend, J. T., \& Ashby, F. G. (1983). Stochastic modelling of elementary psychological processes. New York: Cambridge University Press.

Walton, M., \& SPEnCE, C. (2004). Cross-modal congruency and visual capture in a visual elevation discrimination task. Experimental Brain Research, 154, 113-120.

Yamamoto, S., \& KitaZAWA, S. (2001). Reversal of subjective temporal order due to arm crossing. Nature Neuroscience, 4, 759-765.

YANTIS S. (1996). Attentional capture in vision. In A. F. Kramer, M. G. Coles, \& G. D. Logan (Eds.), Converging operations in the study of visual selective attention (pp. 45-76). Washington, DC: American Psychological Association.

YANTIS, S. (2000). Goal-directed and stimulus-driven determinants of attentional control. In S. Monsell \& J. Driver (Eds.), Control of cognitive processes: Attention and performance XVIII (pp. 73-103). Cambridge, MA: MIT Press.

\section{NOTES}

1. To investigate the potential speed-accuracy tradeoff for the fixed hand further, we calculated inverse efficiency (IE) scores for the interaction between posture and stimulated hand. IE scores are a standard way to combine RT and accuracy data into a single score, computed as the mean RT divided by the proportion of correct trials in a given condition (e.g., Townsend \& Ashby, 1983); a higher IE value indicates worse performance, just as for RT or accuracy measures. Analysis of IE scores confirmed a significant effect of posture change when the vibrotactile target was presented to the mobile hand (hands close, $M=183 \mathrm{msec}$, $S D=62$; hands far, $M=61 \mathrm{msec}, S D=19 ; p<.0005$ on paired sample $t$ test) but no effect of posture change when it was presented to the fixed hand (hands close, $M=158 \mathrm{msec}, S D=141$; hands far, $M=$ $185 \mathrm{msec}, S D=44 ; p=.4$ on paired sample $t$ test), thus further supporting the conclusions from our main analyses.

2. Ten new participants completed four blocks of 140 experimental trials in the crossed-hands posture of Experiment 3. Additional foam cubes were placed on the tabletop in front of the participant (to support the upper arm) in order to prevent him or her from receiving tactile cues regarding the crossed posture of the arms attributable to the forearms touching each other. The ambient lights in the experimental testing room were switched on for half of the blocks of trials (as in Experiments 1-3) and were switched off for the remainder of the blocks, with the order of presentation of the lights-on and lights-off blocks counterbalanced across participants. The experimental design was, in all other respects, similar to that described in Experiment 3. A within-subjects ANOVA conducted on the mean cross-modal congruency effects, with the factors of hand visibility (2) and relative distractor hemifield (2), revealed a significant main effect of relative distractor hemifield in both the RT $[F(1,9)=34.8, p<.0001]$ and the error $[F(1,9)=7.5, p<.02]$ data. This was caused by larger cross-modal congruency effects being reported when the visual distractors were presented from the same hemifield as the vibrotactile targets ( $M=159 \mathrm{msec}$ and $10.3 \%$, respectively) than when they were presented from different hemifields ( $M=69 \mathrm{msec}$ and $6.4 \%$ ). Crucially, however, there was no main effect of hand visibility or any interaction between hand visibility and relative distractor hemifield in either analysis (all $F \mathrm{~S}<2.1$ ), thus demonstrating that visual cues played no significant role in the remapping of visuotactile space seen in Experiment 3. 


\section{APPENDIX}

Support for the existence of a perceptual component to the visual-tactile cross-modal congruency effect comes from the results of a further experiment in which participants $(N=5)$ were required to make unspeeded discrimination responses regarding the elevation of the vibrotactile targets. Using an experimental design very similar to that of the unpredictable target-side blocks in Experiment 1, but including an additional, neutral condition in which vibrotactile targets were presented in the absence of any visual distractors (the participants performed one block of 20 practice trials, followed by four blocks of 100 experimental trials, and the visual distractors, when present, led the vibrotactile targets by $30 \mathrm{msec}$ ), we found that even when the participants were prevented from responding until at least $1,000 \mathrm{msec}$ after the onset of the vibrotactile target, and when the accuracy (rather than the speed) of their responding was strongly stressed, the participants still made some errors when trying to localize the vibrotactile targets.

Crucially, these errors were more prevalent when the vibrotactile target and the visual distractor were presented from incongruent locations on the same side/hand (i.e., from the same foam block) than when they were presented from incongruent elevations on different sides/hands (i.e., from different foam blocks). (Note that the visual distractor could not have ventriloquized the perceived location of the vibrotactile target on congruent same-side/hand trials, because the two stimuli were al- ready presented from virtually the same position.) A one-way within-subjects ANOVA on the arcsin-transformed proportions of errors $(2 \arcsin \sqrt{ } \mathrm{x}$; Howell, 1999), with the factor of visual distractor position (five levels), supported this result statistically $[F(4,16)=3.74, p<.03]$. The percentage of errors when the visual distractors were presented from an incongruent location on the same side/hand as the target $(M=5.7 \%)$ was significantly higher than that for any other condition (mean error rate for the congruent same side $=1.8 \%$, for the congruent different sides $=1.6 \%$, for incongruent different sides $=1.1 \%$, and for the no-distractor condition $=0.8 \%$; all $p \mathrm{~s}<.04$, by Fisher's LSD post hoc test). But note that this cost for unspeeded accuracy, on same-side incongruent trials, appears to be smaller than the typical crossmodal congruency effect found in the speeded tasks of our main experiments and was found only for same-side trials, suggesting that response competition may also play a role in the speeded experiments.

These results would, therefore, appear to show a perceptual interaction between the perceived locations of the vibrotactile target and the visual distractor stimuli, at least on incongruent sameside/hand trials. This spatial ventriloquism of the apparent location of the vibrotactile stimulus toward the location of the visual distractor (see also Pick, Warren, \& Hay, 1969) supports the view that at least some component of the cross-modal congruency effect may have a perceptual origin (see also Marks, 2004, on this point). 